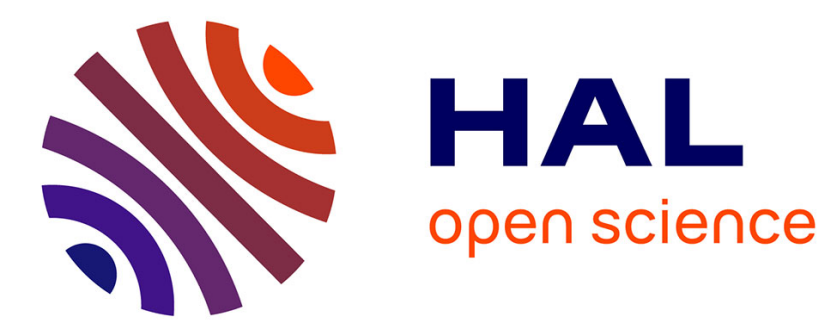

\title{
Mortar spectral element discretization of the Stokes problem in axisymmetric domains
}

Saloua Mani Aouadi, Christine Bernardi, Jamil Satouri

\section{To cite this version:}

Saloua Mani Aouadi, Christine Bernardi, Jamil Satouri. Mortar spectral element discretization of the Stokes problem in axisymmetric domains. Numerical Methods for Partial Differential Equations, 2014, Volume 30 (Issue 1), pp.44-73. hal-00564003

\section{HAL Id: hal-00564003 \\ https://hal.science/hal-00564003}

Submitted on 7 Feb 2011

HAL is a multi-disciplinary open access archive for the deposit and dissemination of scientific research documents, whether they are published or not. The documents may come from teaching and research institutions in France or abroad, or from public or private research centers.
L'archive ouverte pluridisciplinaire HAL, est destinée au dépôt et à la diffusion de documents scientifiques de niveau recherche, publiés ou non, émanant des établissements d'enseignement et de recherche français ou étrangers, des laboratoires publics ou privés.

$$
\text { Copyright }
$$




\title{
Mortar spectral element discretization of the Stokes problem in axisymmetric domains
}

\author{
Saloua Mani Aouadi ${ }^{1}$, Christine Bernardi ${ }^{2}$, and Jamil Satouri ${ }^{1}$
}

\begin{abstract}
The Stokes problem in a tridimensional axisymmetric domain results into a countable family of two-dimensional problems when using the Fourier coefficients with respect to the angular variable. Relying on this dimension reduction, we propose and study a mortar spectral element discretization of the problem. Numerical experiments confirm the efficiency of this method.
\end{abstract}

\section{Résumé}

L'utilisation des coefficients de Fourier par rapport à la variable angulaire permet de réduire le problème de Stokes dans un ouvert tridimensionnel axisymétrique à une famille dénombrable de problèmes bidimensionnels. Grâce à cette réduction de dimension, nous proposons une discrétisation de ce problème par la méthode d'éléments spectraux avec joints et nous en effectuons l'analyse numérique. Des expériences numériques confirment l'intérêt de cette méthode.

Key words: Axisymmetric domains, mortar method, spectral element method, Stokes equation.

\footnotetext{
${ }^{1}$ Faculty of Sciences of Tunis, University of Tunis El Manar, 2060 Tunis, Tunisia.

${ }^{2}$ Laboratoire Jacques-Louis Lions, C.N.R.S. \& Université Pierre et Marie Curie, Boîte courrier 187, 4 place Jussieu, 75252 Paris Cedex 05, France.

e-mails: saloua.mani@fst.rnu.tn, bernardi@ann.jussieu.fr, jamil.satouri@yahoo.fr
} 



\section{Introduction}

The Stokes system

$$
\left\{\begin{array}{lr}
-\triangle \breve{\boldsymbol{u}}+\operatorname{grad} \breve{p}=\breve{\boldsymbol{f}} & \text { in } \breve{\Omega}, \\
\operatorname{div} \breve{\boldsymbol{u}}=0 & \text { in } \breve{\Omega}, \\
\breve{\boldsymbol{u}}=\breve{\boldsymbol{g}} & \text { on } \partial \breve{\Omega},
\end{array}\right.
$$

models the laminar flow of a viscous incompressible fluid in a domain $\breve{\Omega}$, when subjected to a density of forces $\breve{\boldsymbol{f}}$ and with boundary data $\breve{\boldsymbol{g}}$, the unknowns being the velocity $\breve{\boldsymbol{u}}$ and the pressure $\breve{p}$ of the fluid. We are specifically interested in the case where $\breve{\Omega}$ is tri-dimensional and axisymmetric, i.e. invariant by rotation around an axis. Note that this type of geometry appears in a large number of realistic situations, for instance for the flow in a cylindrical pipe or around a spherical obstacle.

The main idea for handling three-dimensional problems in such geometries consists in using the Fourier coefficients of the data and the solution with respect to the angular variable: Indeed, the three-dimensional problem is then reduced to a countable family of uncoupled two-dimensional problems, one for each Fourier coefficient, in the meridian domain. The drawback is that the variational formulation of each problem involves weighted Sobolev spaces, as fully investigated in [2] in a general framework.

The discretization is then performed in two steps. First, we use Fourier truncation, i.e. we only solve a finite number of two-dimensional problems and recall the estimate of the corresponding error from [2, Thm IX.1.9]. Second, we consider a discretization of each two-dimensional problem. Even if finite element discretizations have already been studied in this context, see [4] for instance, we have chosen here to use spectral type methods in order to preserve the accuracy of Fourier truncation. More precisely, in order to handle the possible complexity of the two-dimensional domain $\Omega$, we consider a mortar spectral element discretization of each problem. Indeed, handling such geometries is one of the first applications of the mortar element method as introduced in [7]. We prove the well-posedness of each discrete problem and also establish optimal error estimates (see [11] for the first results in this direction). We present some numerical experiments which confirm the interest of this discretization.

The outline of the paper is as follows:

- In Section 2, we recall from [2] the variational formulation of the twodimensional problems and also the error issued from Fourier truncation.

- In Section 3, we describe the discrete problems constructed from the mortar spectral element method and we prove their well-posedness.

- Section 4 is devoted to the numerical analysis of these problems.

- Numerical experiments are presented in Section 5. 


\section{The two-dimensional problems}

We first make precise some notation about the geometry of the axisymmetric domain and introduce the weighted spaces which are needed on the twodimensional domain. Next we write the variational formulation of the twodimensional problems and recall their well-posedness. We conclude with an estimate for the error due to Fourier truncation.

\subsection{About the geometry}

With a point in $\mathbb{R}^{3}$, we associate its Cartesian coordinates $(x, y, z)$ and its cylindrical ones $(r, \theta, z)$ with

$$
x=r \cos \theta, y=r \sin \theta, \quad r \in \mathbb{R}_{+}, \theta \in[-\pi, \pi[.
$$

We denote by $\mathbb{R}_{+}^{2}$ the product $\mathbb{R}_{+} \times \mathbb{R}$ and consider a polygon $\Omega$ in $\mathbb{R}_{+}^{2}$ with boundary $\partial \Omega$ made of a finite number of segments $\Gamma_{i}, 1 \leq i \leq I$. The endpoints of these segments are known as corners of $\Omega$ : We call $c_{1}, c_{2}, \ldots c_{p}$ the corners of the polygon which are on the axis $r=0$, and $e_{1}, e_{2}, \ldots e_{j}$ the other corners of $\Omega$. Let $\Gamma_{0}$ be the intersection of $\partial \Omega$ with the axis $r=0$ and $\Gamma=\partial \Omega \backslash \Gamma_{0}$.

Let $\breve{\Omega}$ be the domain of $\mathbb{R}^{3}$ obtained by rotation of $\Omega$ around the axis $r=0$. The set $\Omega$ is then called meridian domain and we have

$$
\breve{\Omega}=\left\{(r, \theta, z) \in \mathbb{R}^{3}, \quad(r, z) \in \Omega \cup \Gamma_{0},-\pi \leq \theta<\pi\right\} .
$$

In Figure 1, we illustrate some examples of domains $\breve{\Omega}$ which we treat in our numerical experiments.
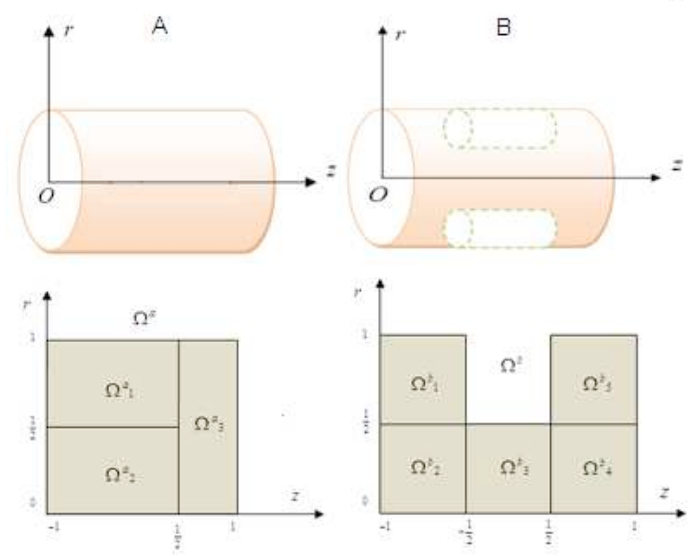

Figure 1: Examples of domains $\Omega$ and $\breve{\Omega}$ 


\subsection{Weighted Sobolev spaces}

We define the Hilbert spaces $L_{1}^{2}(\Omega), L_{-1}^{2}(\Omega)$ and $H_{1}^{m}(\Omega)$, for any positive integer $m$ by:

$$
\begin{gathered}
L_{ \pm 1}^{2}(\Omega)=\{v: \Omega \longrightarrow \mathbb{C} \text { measurable; } \\
\left.\qquad v \|_{L_{ \pm 1}^{2}(\Omega)}=\left(\int_{\Omega}\left|v^{2}(r, z)\right| r^{ \pm 1} d r d z\right)^{\frac{1}{2}}<+\infty\right\} \\
H_{1}^{m}(\Omega)=\left\{v \in L_{1}^{2}(\Omega) ;\|v\|_{H_{1}^{m}(\Omega)}=\left(\sum_{k=0}^{m} \sum_{\ell=0}^{k}\left\|\partial_{r}^{\ell} \partial_{z}^{k-\ell} v\right\|_{L_{1}^{2}(\Omega)}^{2}\right)^{\frac{1}{2}}<+\infty\right\} .
\end{gathered}
$$

For a positive real number $s$, the space $H_{1}^{s}(\Omega)$ is deduced in a standard way by interpolation between the space $H_{1}^{[s]}(\Omega)$ and $H_{1}^{[s]+1}(\Omega)$, where $[s]$ stands for the integral part of $s$. We also need the Hilbert space $V_{1}^{1}(\Omega)=H_{1}^{1}(\Omega) \cap L_{-1}^{2}(\Omega)$, and we provide it with the norm

$$
\|w\|_{V_{1}^{1}(\Omega)}=\left(\|w\|_{H_{1}^{1}(\Omega)}^{2}+\|w\|_{L_{-1}^{2}(\Omega)}^{2}\right)^{\frac{1}{2}}
$$

Remark 2.1 In the monodimensional case of an edge $\Lambda$ of $\Omega$, the spaces $L_{ \pm 1}^{2}(\Lambda)$, $H_{1}^{s}(\Lambda)$ and $V_{1}^{1}(\Lambda)$ are defined in the same way as in the two-dimensional case by using the measure $d \tau=r d r$ if $\Lambda$ is perpendicular to the axis $(O z)$ and $d \tau=d z$ if it is parallel to this axis. For more details see [2, Chap. II].

Indeed, with any scalar function $\breve{v}$ in $L^{2}(\breve{\Omega})$, we associate its Fourier coefficients $v^{k}, k \in \mathbb{Z}$, given by

$$
v^{k}(r, z)=\frac{1}{\sqrt{2 \pi}} \int_{-\pi}^{\pi} \breve{v}(r, \theta, z) e^{-i k \theta} d \theta
$$

It is readily checked that each $v^{k}$ then belongs to $L_{1}^{2}(\Omega)$.

Similarly, for each vector field $\breve{\boldsymbol{v}}$ in $L^{2}(\breve{\Omega})^{3}$, we consider its cylindrical componenta $\breve{v}_{r}, \breve{v}_{\theta}$ and $\breve{v}_{z}$ and the associated Fourier coefficient $\left(v_{r}^{k}, v_{\theta}^{k}, v_{z}^{k}\right)$ defined by the analogue of $(2.1)$ which now belong to $L_{1}^{2}(\Omega)^{3}$. It is proved in [2, Thm II.3.6] that the Fourier transform: $\breve{\boldsymbol{v}} \rightarrow\left(v_{r}^{k}, v_{\theta}^{k}, v_{z}^{k}\right)_{k}$ maps $H^{1}(\breve{\Omega})^{3}$ onto $\prod_{k \in \mathbb{Z}} \boldsymbol{H}_{(k)}^{1}(\Omega)$ with

$$
\boldsymbol{H}_{(k)}^{1}(\Omega)=\left\{\begin{array}{lr}
V_{1}^{1}(\Omega) \times V_{1}^{1}(\Omega) \times H_{1}^{1}(\Omega) & \text { if } k=0, \\
\left\{\left(v_{r}, v_{\theta}, v_{z}\right) \in H_{1}^{1}(\Omega) \times H_{1}^{1}(\Omega) \times V_{1}^{1}(\Omega) ; v_{r}+i k v_{\theta} \in L_{-1}^{2}(\Omega)\right\} & \text { if }|k|=1, \\
V_{1}^{1}(\Omega) \times V_{1}^{1}(\Omega) \times V_{1}^{1}(\Omega) & \text { if }|k| \geq 2 .
\end{array}\right.
$$

More general results exist for the spaces $H^{s}(\breve{\Omega})^{3}$, see [2, Chap. II], we do not state them for simplicity. 


\subsection{Variational formulation of the problems}

In order to take into account the boundary conditions, we introduce the spaces

$$
\begin{aligned}
H_{1 \diamond}^{1}(\Omega)=\left\{v \in H_{1}^{1}(\Omega) ; v=0 \text { on } \Gamma\right\}, & \\
& V_{1 \diamond}^{1}(\Omega)=V_{1}^{1}(\Omega) \cap H_{1 \diamond}^{1}(\Omega), \quad \boldsymbol{H}_{(k) \diamond}^{1}(\Omega)=\boldsymbol{H}_{(k)}^{1}(\Omega) \cap H_{1 \diamond}^{1}(\Omega)^{3} .
\end{aligned}
$$

We also need the spaces

$$
L_{(k)}^{2}(\Omega)= \begin{cases}\left\{q \in L_{1}^{2}(\Omega) ; \int_{\Omega} q(r, z) r d r d z=0\right\} & \text { if } k=0, \\ L_{1}^{2}(\Omega) & \text { if }|k| \geq 1 .\end{cases}
$$

We use a lifting of the boundary data $\breve{\boldsymbol{g}}$ that we still denote by $\breve{\boldsymbol{g}}$ for simplicity.

Then, it is readily checked that, if $(\breve{\boldsymbol{u}}, \breve{p})$ is the solution of problem (1.1) with data $(\breve{\boldsymbol{f}}, \breve{\boldsymbol{g}})$ in $L^{2}(\breve{\Omega})^{3} \times H^{1}(\breve{\Omega})^{3}$, the Fourier coefficients $\left(\boldsymbol{u}^{k}=\left(u_{r}^{k}, u_{\theta}^{k}, u_{z}^{k}\right), p^{k}\right)$ are the solutions of the following variational problems, for all $k \in \mathbb{Z}$ :

Find $\left(\boldsymbol{u}^{k}, p^{k}\right)$ in $\boldsymbol{H}_{(k)}^{1}(\Omega) \times L_{(k)}^{2}(\Omega)$, with $\boldsymbol{u}^{k}-\boldsymbol{g}^{k}$ in $\boldsymbol{H}_{(k) \diamond}^{1}(\Omega)$, such that

$$
\begin{gathered}
\forall \boldsymbol{v} \in \boldsymbol{H}_{(k) \diamond}^{1}(\Omega), \quad \mathcal{A}_{k}\left(\boldsymbol{u}^{k}, \boldsymbol{v}\right)+\mathcal{B}_{k}\left(\boldsymbol{v}, p^{k}\right)=\left\langle\boldsymbol{f}^{k}, \boldsymbol{v}\right\rangle, \\
\forall q \in L_{1}^{2}(\Omega), \quad \mathcal{B}_{k}\left(\boldsymbol{u}^{k}, q\right)=0,
\end{gathered}
$$

where the rather complex sesquilinear forms $\mathcal{A}_{k}(\cdot, \cdot)$ and $\mathcal{B}_{k}(\cdot, \cdot)$ are defined by

$$
\begin{aligned}
& \mathcal{A}_{k}(\boldsymbol{u}, \boldsymbol{w})=a_{0}\left(u_{r}, w_{r}\right)+a_{0}\left(u_{\theta}, w_{\theta}\right)+a_{0}\left(u_{z}, w_{z}\right) \\
& \quad+\int_{\Omega}\left(\frac{1+k^{2}}{r^{2}}\left(u_{r} \bar{w}_{r}+u_{\theta} \bar{w}_{\theta}\right)+\frac{2 i k}{r^{2}}\left(u_{\theta} \bar{w}_{r}-u_{r} \bar{w}_{\theta}\right)+\frac{k^{2}}{r^{2}} u_{z} \bar{w}_{z}\right) r d r d z,
\end{aligned}
$$

with

$$
a_{0}(u, w)=\int_{\Omega}\left(\partial_{r} u \partial_{r} \bar{w}+\partial_{z} u \partial_{z} \bar{w}\right)(r, z) r d r d z
$$

and

$$
\mathcal{B}_{k}(\boldsymbol{w}, q)=-\int_{\Omega} q\left(\partial_{r} \bar{w}_{r}+\frac{1}{r}\left(\overline{w_{r}+i k w_{\theta}}\right)+\partial_{z} \bar{w}_{z}\right) r d r d z
$$

The Hermitian product $\langle\cdot, \cdot\rangle$ is given by

$$
\langle\boldsymbol{f}, \boldsymbol{v}\rangle=\int_{\Omega} \boldsymbol{f}(r, z) \cdot \overline{\boldsymbol{v}}(r, z) r d r d z .
$$

From now on, the space $\boldsymbol{H}_{(k)}^{1}(\Omega)$ is equipped with the norm

$$
\|\boldsymbol{v}\|_{\boldsymbol{H}_{(k)}^{1}(\Omega)}=\mathcal{A}_{k}(\boldsymbol{v}, \boldsymbol{v})^{\frac{1}{2}}
$$

(indeed, the quantity $\mathcal{A}_{k}(\boldsymbol{v}, \boldsymbol{v})$ is real and nonnegative) and the space $L_{(k)}^{2}(\Omega)$ is equipped with the norm $\|\cdot\|_{L_{1}^{2}(\Omega)}$. Then, appropriate properties of the forms $\mathcal{A}_{k}(\cdot, \cdot)$ and $\mathcal{B}_{k}(\cdot, \cdot)$ on these spaces are derived in [2, Prop. IX.1.3], which leads to the following result. 
Proposition 2.2 For any data $\boldsymbol{f}^{k}$ in $L_{1}^{2}(\Omega)^{3}$ and $\boldsymbol{g}^{k}$ in $\boldsymbol{H}_{(k)}^{1}(\Omega)$ satisfying moreover in the case $k=0$ the null flux condition

$$
\int_{\Gamma}\left(g_{r} n_{r}+g_{z} n_{z}\right)(\tau) r(\tau) d \tau=0
$$

problem (2.2) has a unique solution $\left(\boldsymbol{u}^{k}, p^{k}\right)$ in $\boldsymbol{H}_{(k)}^{1}(\Omega) \times L_{(k)}^{2}(\Omega)$. Moreover, this solution satisfies, for a constant $c$ only depending on $\Omega$,

$$
\left\|\boldsymbol{u}^{k}\right\|_{\boldsymbol{H}_{(k)}^{1}(\Omega)}+\left\|p^{k}\right\|_{L_{1}^{2}(\Omega)} \leq c\left(\left\|\boldsymbol{f}^{k}\right\|_{L_{1}^{2}(\Omega)^{3}}+\left\|\boldsymbol{g}^{k}\right\|_{\boldsymbol{H}_{(k)}^{1}(\Omega)}\right)
$$

Remark 2.3 The data $\breve{\boldsymbol{f}}$ and $\breve{\boldsymbol{g}}$ are said to be axisymmetric if all functions $\breve{f}_{r}$, $\breve{f}_{\theta}$ and $\breve{f}_{z}, \breve{g}_{r}, \breve{g}_{\theta}$ and $\breve{g}_{z}$ are independent of $\theta$. In this case, only problem (2.2) for $k=0$ has a non-zero solution. Moreover it results into the set of two uncoupled problems

Find $u_{\theta}$ in $V_{1}^{1}(\Omega)$, with $u_{\theta}-g_{\theta}$ in $V_{1 \diamond}^{1}(\Omega)$, such that

$$
\forall v \in V_{1 \diamond}^{1}(\Omega), \quad a_{1}\left(u_{\theta}, v\right)=\left\langle f_{\theta}, v\right\rangle
$$

and

$$
\begin{aligned}
& \text { Find }\left(u_{r}, u_{z}, p\right) \text { in } V_{1}^{1}(\Omega) \times H_{1}^{1}(\Omega) \times L_{(0)}^{2}(\Omega) \text {, } \\
& \text { with } u_{r}-g_{r} \text { in } V_{1 \diamond}^{1}(\Omega) \text { and } u_{z}-g_{z} \text { in } H_{1 \diamond}^{1}(\Omega) \text {, such that } \\
& \forall\left(v_{r}, v_{z}\right) \in V_{1 \diamond}^{1}(\Omega) \times H_{1 \diamond}^{1}(\Omega), \\
& a_{1}\left(u_{r}, v_{r}\right)+a_{0}\left(u_{z}, v_{z}\right)+b\left(v_{r}, v_{z} ; p\right)=\left\langle f_{r}, v_{r}\right\rangle+\left\langle f_{z}, v_{z}\right\rangle, \\
& \forall q \in L_{(0)}^{2}(\Omega), b\left(u_{r}, u_{z} ; q\right)=0,
\end{aligned}
$$

where the sesquilinear forms $a_{1}(\cdot, \cdot)$ and $b(\cdot, \cdot)$ are now defined by

$$
\begin{aligned}
& a_{1}(u, w)=a_{0}(u, w)+\int_{\Omega} u(r, z) \bar{w}(r, z) r^{-1} d r d z, \\
& b(\boldsymbol{w}, q)=-\int_{\Omega} q\left(\partial_{r} \bar{w}_{r}+\frac{1}{r} \overline{w_{r}}+\partial_{z} \bar{w}_{z}\right) r d r d z .
\end{aligned}
$$

These problems seem much simpler and their discretization is considered separately in the next section.

\subsection{Fourier truncation}

Of course, we intend to discretize only a finite number of problems (2.2). So, we chose a positive integer $K$ and, in analogy with the formula

$$
\breve{\boldsymbol{u}}(r, \theta, z)=\frac{1}{\sqrt{2 \pi}} \sum_{k \in \mathbb{Z}} \boldsymbol{u}^{k}(r, z) e^{i k \theta}, \quad \breve{p}(r, \theta, z)=\frac{1}{\sqrt{2 \pi}} \sum_{k \in \mathbb{Z}} p^{k}(r, z) e^{i k \theta}
$$


we define an approximation of the solution $(\breve{\boldsymbol{u}}, \breve{p})$ of problem (1.1) by

$$
\begin{aligned}
\breve{\boldsymbol{u}}_{K}(r, \theta, z)=\frac{1}{\sqrt{2 \pi}} \sum_{|k| \leq K} \boldsymbol{u}^{k}(r, z) e^{i k \theta} & \\
\breve{p}_{K}(r, \theta, z) & =\frac{1}{\sqrt{2 \pi}} \sum_{|k| \leq K} p^{k}(r, z) e^{i k \theta} .
\end{aligned}
$$

Indeed, the following result can be found in [2, Thm IX.1.9].

Proposition 2.4 For any $s \geq 0$, if the data $(\breve{\boldsymbol{f}}, \breve{\boldsymbol{g}})$ belong to $H^{s-1}(\breve{\Omega})^{3} \times$ $H^{s+1}(\Omega)^{3}$, the following estimate holds between the solution $(\breve{\boldsymbol{u}}, \breve{p})$ of problem (1.1) and its approximation $\left(\breve{\boldsymbol{u}}_{K}, \breve{p}_{K}\right)$ defined in (2.7)

$$
\left\|\breve{\boldsymbol{u}}-\breve{\boldsymbol{u}}_{K}\right\|_{H^{1}(\breve{\Omega})^{3}}+\left\|\breve{p}-\breve{p}_{K}\right\|_{L^{2}(\breve{\Omega})} \leq c K^{-s}\left(\|\breve{\boldsymbol{f}}\|_{H^{s-1}(\breve{\Omega})^{3}}+\|\breve{\boldsymbol{g}}\|_{H^{s+1}(\breve{\Omega})^{3}}\right) .
$$

Remark 2.5 When Fourier truncation is used, the Fourier coefficients of the data $\boldsymbol{f}^{k}$ and $\boldsymbol{g}^{k},|k| \leq K$, are usually computed by a quadrature formula: With $\theta_{m}=\frac{2 m \pi}{2 K+1}$, the approximate Fourier coefficients are given for $|k| \leq K$ by

$$
\begin{aligned}
\boldsymbol{f}^{k *}(r, z)=\frac{\sqrt{2 \pi}}{2 K+1} \sum_{|m| \leq K} \breve{\boldsymbol{f}}\left(r, \theta_{m}, z\right) e^{-i k \theta_{m}} & \\
\boldsymbol{g}^{k *}(r, z) & =\frac{\sqrt{2 \pi}}{2 K+1} \sum_{|m| \leq K} \breve{\boldsymbol{g}}\left(r, \theta_{m}, z\right) e^{-i k \theta_{m}} .
\end{aligned}
$$

We do not take this modification into account in the next section, since the final error estimates are exactly the same. 


\section{The discrete problems}

We first recall the decomposition of the domain and the approximation spaces that are required for the mortar spectral element method. Next, we write the corresponding discrete problems, first in the case of axiysmmetric data, second in the general case, and prove their well-posedness.

\subsection{About the mortar element method}

In view of the discretization, we consider a decomposition of $\Omega$ into $L$ open rectangles $\Omega_{\ell}, 1 \leq \ell \leq L$, such that

$$
\bar{\Omega}=\bigcup_{\ell=1}^{L} \bar{\Omega}_{\ell} \quad \text { and } \quad \Omega_{\ell} \cap \Omega_{m}=\varnothing, \quad 1 \leq \ell<m \leq L .
$$

Note that the edges of the $\Omega_{\ell}$ are either parallel or orthogonal to the axis $(O z)$.

For any two-dimensional domain $\mathcal{O}$ and nonnegative integer $N, \mathbb{P}_{N}(\mathcal{O})$ stands for the space of restrictions to $\mathcal{O}$ of polynomials on $\mathbb{R}^{2}$ with degree $\leq N$ with respect to each variable $r$ and $z$. In view of the discretization, we define a $L$-tuple of positive integers $\delta=\left(N_{1}, \ldots, N_{L}\right)$. Indeed, the idea of the mortar spectral element method is to approximate the discrete solutions in a subspace of

$$
\mathbb{Y}_{\delta}(\Omega)=\left\{v_{\delta} \in L_{1}^{2}(\Omega) ;\left.v_{\delta}\right|_{\Omega_{\ell}} \in \mathbb{P}_{N_{\ell}}\left(\Omega_{\ell}\right), 1 \leq \ell \leq L\right\} .
$$

Let also $\mathbb{Y}_{\delta}^{\diamond}(\Omega)$ stand for the space of functions in $\mathbb{Y}_{\delta}(\Omega)$ vanishing on $\Gamma$.

To define this subspace, we introduce the skeleton $\mathcal{S}$ of the domain decomposition, equal to $\bigcup_{\ell=1}^{L} \partial \Omega_{\ell} \backslash \partial \Omega$. It admits a partition without overlap into mortars

$$
\overline{\mathcal{S}}=\bigcup_{\mu=1}^{M^{+}} \gamma_{\mu}^{+}, \quad \text { with } \quad \gamma_{\mu}^{+} \cap \gamma_{\mu^{\prime}}^{+}=\varnothing, \quad 1 \leq \mu<\mu^{\prime} \leq M^{+}
$$

each $\gamma_{\mu}^{+}$being a whole edge of one of $\Omega_{\ell}$, which is then denoted by $\Omega_{\mu}^{+}$. Note that the choice of this decomposition is not unique, however it is decided a priori for all the discretizations we work with. Once it is fixed, we have another partition of the skeleton into non-mortars:

$$
\overline{\mathcal{S}}=\bigcup_{m=1}^{M^{-}} \gamma_{m}^{-}, \quad \text { with } \quad \gamma_{m}^{-} \cap \gamma_{m^{\prime}}^{-}=\varnothing, \quad 1 \leq m<m^{\prime} \leq M^{-},
$$

where each $\gamma_{m}^{-}$is a whole edge of one of $\Omega_{\ell}$, then denoted by $\Omega_{m}^{-}$(if there exists an index $\mu$ such that $\gamma_{m}^{-}$and $\gamma_{\mu}^{+}$coincide, $\Omega_{m}^{-}$is different of $\Omega_{\mu}^{+}$).

Next, with all $v_{\delta}$ in $\mathbb{Y}_{\delta}(\Omega)$, we associate the mortar function $\phi_{v_{\delta}}$ in $L_{1}^{2}(\mathcal{S})$ defined by $\left.\phi_{v_{\delta}}\right|_{\gamma_{\mu}^{+}}=\left.\left(\left.v_{\delta}\right|_{\Omega_{\mu}^{+}}\right)\right|_{\gamma_{\mu}^{+}}, 1 \leq \mu \leq M^{+}$. With obvious definition of $N_{m}^{-}$, we 
define our fundamental discrete space $\mathbb{X}_{\delta}$ by:

$$
\begin{aligned}
\mathbb{X}_{\delta}(\Omega) & =\left\{v_{\delta} \in \mathbb{Y}_{\delta} ;\right. \\
\forall \psi & \left.\in \mathbb{P}_{N_{m}^{-}-2}\left(\gamma_{m}^{-}\right), \int_{\gamma_{m}^{-}}\left(\left.v_{\delta}\right|_{\Omega_{m}^{-}}-\phi_{v_{\delta}}\right)(\tau) \psi(\tau) d \tau=0,1 \leq m \leq M^{-}\right\}
\end{aligned}
$$

with $d \tau=r d r$ if the non-mortar $\gamma_{m}^{-}$is parallel to the axis $(O r)$ and $d \tau=d z$ if $\gamma_{m}^{-}$is parallel to the axis $(O z)$. We also need its subspaces defined as follows:

(i) $\mathbb{X}_{\delta}^{\diamond}$ is the space of functions $v_{\delta}$ vanishing on $\Gamma$;

(ii) $\mathbb{X}_{\delta}^{*}$ is the space

$$
\mathbb{X}_{\delta}^{*}(\Omega)=\left\{v_{\delta} \in \mathbb{X}_{\delta}(\Omega) ; v_{\ell}=\left.v_{\delta}\right|_{\Omega_{\ell}} \in L_{-1}^{2}\left(\Omega_{\ell}\right), 1 \leq \ell \leq L\right\}
$$

and $\mathbb{X}_{\delta}^{0}$ is the intersection of $\mathbb{X}_{\delta}^{\diamond}$ and $\mathbb{X}_{\delta}^{*}$.

Finally, we introduce the space

$$
\mathbb{X}_{\delta(k)}(\Omega)=\left\{\begin{array}{lr}
\mathbb{X}_{\delta}^{*}(\Omega) \times \mathbb{X}_{\delta}^{*}(\Omega) \times \mathbb{X}_{\delta}(\Omega) & \text { if } k=0, \\
\left\{\left(v_{r}, v_{\theta}, v_{z}\right) \in \mathbb{X}_{\delta}(\Omega) \times \mathbb{X}_{\delta}(\Omega) \times \mathbb{X}_{\delta}^{*}(\Omega) ; v_{r}+i k v_{\theta} \in \mathbb{X}_{\delta}^{*}(\Omega)\right\} & \text { if }|k|=1, \\
\mathbb{X}_{\delta}^{*}(\Omega) \times \mathbb{X}_{\delta}^{*}(\Omega) \times \mathbb{X}_{\delta}^{*}(\Omega) & \text { if }|k| \geq 2,
\end{array}\right.
$$

and its intersection $\mathbb{X}_{\delta(k)}^{\diamond}(\Omega)$ with $\mathbb{X}_{\delta}^{\diamond}(\Omega)^{3}$. All these spaces are needed for the approximation of the velocity.

The spaces for the approximation of the pressure are more simpler, they are defined by

$$
\begin{aligned}
& \mathbb{M}_{\delta}(\Omega)=\left\{v_{\delta} \in L_{1}^{2}(\Omega) ;\left.v_{\delta}\right|_{\Omega_{\ell}} \in \mathbb{P}_{N_{\ell}-2}\left(\Omega_{\ell}\right), 1 \leq \ell \leq L\right\} \\
& \mathbb{M}_{\delta(k)}(\Omega)=\mathbb{M}_{\delta}(\Omega) \cap L_{(k)}^{2}(\Omega) .
\end{aligned}
$$

To conclude, we introduce quadrature formulas. Let $\left(\xi_{j}, \rho_{j}\right), 0 \leq j \leq N$, denote the nodes and weights of the Gauss-Lobatto quadrature formula on $[-1,1]$ for the measure $d \zeta$ and $\left(\zeta_{i}, \omega_{i}\right), 1 \leq i \leq N+1$, their analogues for the measure $(1+\zeta) d \zeta$, see [2, Section VI.1] for a more explicit definition. We denote by $\left(\Omega_{\ell}\right)_{1 \leq \ell \leq L_{0}}$ the rectangles such that $\partial \bar{\Omega}_{\ell} \cap \Gamma_{0} \neq \varnothing$ and by $\left(\Omega_{\ell}\right)_{L_{0}+1 \leq \ell \leq L}$ those such that $\partial \Omega_{\ell} \cap \Gamma_{0}=\varnothing$. If $\Omega_{\ell}$ is equal to $] 0, r_{\ell}^{\prime}[\times] z_{\ell}, z_{\ell}^{\prime}\left[\right.$ for $1 \leq \ell \leq L_{0}$ and to ]$r_{\ell}, r_{\ell}^{\prime}[\times] z_{\ell}, z_{\ell}^{\prime}\left[\right.$ for $L_{0}+1 \leq \ell \leq L$, we use the following definitions:

(i) For $1 \leq \ell \leq L_{0}$ and with $N=N_{\ell}$,

$$
\zeta_{i}^{\ell}=\frac{r_{\ell}^{\prime}}{2}\left(\zeta_{i}+1\right), \quad \omega_{i}^{\ell}=\omega_{i} \frac{r_{\ell}^{\prime 2}}{4}, \quad 1 \leq i \leq N_{\ell}+1
$$


(ii) For $L_{0}+1 \leq \ell \leq L$ and still with $N=N_{\ell}$,

$$
\xi_{i}^{(r) \ell}=\frac{\left(r_{\ell}^{\prime}-r_{\ell}\right)}{2} \xi_{i}+\frac{\left(r_{\ell}^{\prime}+r_{\ell}\right)}{2}, \quad \rho_{i}^{(r) \ell}=\rho_{i} \frac{r_{\ell}^{\prime}-r_{\ell}}{2}, \quad 0 \leq i \leq N_{\ell} ;
$$

(iii) For $1 \leq \ell \leq L$ and once more with $N=N_{\ell}$,

$$
\xi_{j}^{\ell}=\frac{\left(z_{\ell}^{\prime}-z_{\ell}\right)}{2} \xi_{i}+\frac{\left(z_{\ell}^{\prime}+z_{\ell}\right)}{2}, \quad \rho_{j}^{\ell}=\rho_{j} \frac{z_{\ell}^{\prime}-z_{\ell}}{2}, \quad 0 \leq j \leq N_{\ell} .
$$

We are thus in a position to define the discrete scalar product: For all functions $u$ and $v$ such that $u_{\ell}=\left.u\right|_{\Omega_{\ell}}$ and $v_{\ell}=\left.v\right|_{\Omega_{\ell}}$ are continuous on $\bar{\Omega}_{\ell}, 1 \leq \ell \leq L$,

$$
\begin{aligned}
(u, v)_{\delta}=\sum_{\ell=1}^{L_{0}} \sum_{i=1}^{N_{\ell}+1} \sum_{j=0}^{N_{\ell}} u_{\ell}\left(\zeta_{j}^{\ell}, \xi_{i}^{\ell}\right) & v_{\ell}\left(\zeta_{j}^{\ell}, \xi_{i}^{\ell}\right) \omega_{i}^{\ell} \rho_{j}^{\ell} \\
& +\sum_{\ell=L_{0}+1}^{L} \sum_{i=0}^{N_{\ell}} \sum_{j=0}^{N_{\ell}} u_{\ell}\left(\xi_{i}^{(r) \ell}, \xi_{j}^{\ell}\right) v_{\ell}\left(\xi_{i}^{(r) \ell}, \xi_{j}^{\ell}\right) \xi_{i}^{(r) \ell} \rho_{i}^{(r) \ell} \rho_{j}^{\ell} .
\end{aligned}
$$

We denote by $\mathcal{I}_{\ell}^{+}$and $\mathcal{I}_{\ell}$ the Lagrange interpolation operators associated with the nodes $\left(\zeta_{j}^{\ell}, \xi_{i}^{\ell}\right)$ for $1 \leq \ell \leq L_{0}$ and with $\left(\xi_{i}^{(r) \ell}, \xi_{j}^{\ell}\right)$ for $L_{0}+1 \leq \ell \leq L$, respectively, with values in $\mathbb{P}_{N_{\ell}}\left(\Omega_{\ell}\right)$. Let also $\mathcal{I}_{\delta}$ stand for the global interpolation operator with values in $\mathbb{Y}_{\delta}(\Omega)$.

\subsection{The discrete problems for axisymmetric data}

As standard for spectral methods, the discrete problems are constructed by the Galerkin method with numerical integration. The problem associated with (2.5) reads

Find $u_{\theta, \delta}$ in $\mathbb{X}_{\delta}^{*}(\Omega)$, with $u_{\theta, \delta}-\mathcal{I}_{\delta} g_{\theta}$ in $\mathbb{Y}_{\delta}^{\diamond}(\Omega)$, such that

$$
\forall v_{\delta} \in \mathbb{X}_{\delta}^{0}(\Omega), \quad a_{1 \delta}\left(u_{\theta, \delta}, v_{\delta}\right)=\left(f_{\theta}, \bar{v}_{\delta}\right)_{\delta}
$$

where the bilinear form $a_{1 \delta}(\cdot, \cdot)$ is defined by

$$
\begin{aligned}
a_{0 \delta}\left(u_{\delta}, w_{\delta}\right)=\left(\partial_{r} u_{\delta}, \partial_{r} \bar{w}_{\delta}\right)_{\delta}+\left(\partial_{z} u_{\delta}, \partial_{z} \bar{w}_{\delta}\right)_{\delta}, & \\
a_{1 \delta}\left(u_{\delta}, w_{\delta}\right) & =a_{0 \delta}\left(u_{\delta}, w_{\delta}\right)+\left(r^{-1} u_{\delta}, r^{-1} \bar{w}_{\delta}\right)_{\delta} .
\end{aligned}
$$

From now on, we omit the study of this problem and we refer to [1] for its detailed numerical analysis. 
The problem associated with (2.6) reads

Find $\left(u_{r, \delta}, u_{z, \delta}, p_{\delta}\right)$ in $\mathbb{X}_{\delta}^{*}(\Omega) \times \mathbb{X}_{\delta}(\Omega) \times \mathbb{M}_{\delta(0)}(\Omega)$,

with $u_{r, \delta}-\mathcal{I}_{\delta} g_{r}$ in $\mathbb{Y}_{\delta}^{\diamond}(\Omega)$ and $u_{z, \delta}-\mathcal{I}_{\delta} g_{z}$ in $\mathbb{Y}_{\delta}^{\diamond}(\Omega)$, such that

$\forall\left(v_{r, \delta}, v_{z, \delta}\right) \in \mathbb{X}_{\delta}^{0}(\Omega) \times \mathbb{X}_{\delta}^{\diamond}(\Omega)$

$$
\begin{gathered}
a_{1 \delta}\left(u_{r, \delta}, v_{r, \delta}\right)+a_{0 \delta}\left(u_{z, \delta}, v_{z, \delta}\right)+b_{\delta}\left(v_{r, \delta}, v_{z, \delta} ; p_{\delta}\right) \\
=\left(f_{r}, \bar{v}_{r, \delta}\right)_{\delta}+\left(f_{z}, \bar{v}_{z, \delta}\right)_{\delta}, \\
\forall q_{\delta} \in \mathbb{M}_{\delta(0)}(\Omega), \quad b_{\delta}\left(u_{r, \delta}, u_{z, \delta} ; q_{\delta}\right)=0,
\end{gathered}
$$

where the form $b_{\delta}(\cdot, \cdot)$ is defined by

$$
b_{\delta}\left(\boldsymbol{w}_{\delta}, q_{\delta}\right)=-\left(q_{\delta}, \partial_{r} \bar{w}_{r, \delta}+r^{-1} \bar{w}_{r, \delta}+\partial_{z} \bar{w}_{z, \delta}\right)_{\delta} .
$$

In order to investigate the well-posedness of problem (3.7), we now establish some properties of the sesquilinear forms which are involved in it. The continuity of the forms $a_{0 \delta}(\cdot, \cdot)$ and $a_{1 \delta}(\cdot, \cdot)$ follow from the positivity and boundedness of the Gauss-Lobatto formulas, see [6, Remark 13.3] and [2, Lemma VI.1.4]. This also yields the ellipticity of $a_{1 \delta}(\cdot, \cdot)$. However, a further and now well-known argument is needed to prove the ellipticity of $a_{0 \delta}(\cdot, \cdot)$, we refer to [5, Chap IV, Lemma 3.2] for the complete proof. All discrete spaces are equipped with the broken norms that results from their definition, namely

$$
\|v\|_{H_{1 D}^{1}(\Omega)}=\left(\sum_{\ell=1}^{L}\|v\|_{H_{1}^{1}\left(\Omega_{\ell}\right)}^{2}\right)^{\frac{1}{2}}, \quad\|v\|_{V_{1 D}^{1}(\Omega)}=\left(\sum_{\ell=1}^{L}\|v\|_{V_{1}^{1}\left(\Omega_{\ell}\right)}^{2}\right)^{\frac{1}{2}} .
$$

Lemma 3.1 Let $N_{D}$ denote the maximal number of corners of the $\Omega_{\ell}$ which are inside one of the non-mortars $\gamma_{\mu}^{-}, 1 \leq \mu \leq M^{-}$.

(i) The form $a_{1 \delta}(\cdot, \cdot)$ is continuous on $\mathbb{X}_{\delta}^{*}(\Omega) \times \mathbb{X}_{\delta}^{*}(\Omega)$ and elliptic on $\mathbb{X}_{\delta}^{0}(\Omega)$, with norm and ellipticity constant independent of $\delta$.

(ii) If all the $N_{\ell}$ satisfy

$$
N_{\ell} \geq N_{D}+2, \quad 1 \leq \ell \leq L,
$$

the form $a_{0 \delta}(\cdot, \cdot)$ is continuous on $\mathbb{X}_{\delta}(\Omega) \times \mathbb{X}_{\delta}(\Omega)$ and elliptic on $\mathbb{X}_{\delta}^{\diamond}(\Omega)$, with norm and ellipticity constant independent of $\delta$.

Next, we observe that, due to the definition of $\mathbb{M}_{\delta}(\Omega)$, the forms $b(\cdot, \cdot)$ and $b_{\delta}(\cdot, \cdot)$ coincide on $\mathbb{X}_{\delta}^{*}(\Omega) \times \mathbb{X}_{\delta}(\Omega) \times \mathbb{M}_{\delta}(\Omega)$, whence the continuity of $b_{\delta}(\cdot, \cdot)$. However proving the second part of the next lemma is more difficult.

Lemma 3.2 The form $b_{\delta}(\cdot, \cdot)$ is continuous on $\mathbb{X}_{\delta}^{*}(\Omega) \times \mathbb{X}_{\delta}(\Omega) \times \mathbb{M}_{\delta}(\Omega)$, with norm independent of $\delta$. Moreover, for any $q_{\delta} \in \mathbb{M}_{\delta(0)}$, there holds the inf-sup condition

$$
\sup _{\boldsymbol{w}_{\delta} \in \mathbb{X}_{\delta}^{0}(\Omega) \times \mathbb{X}_{\delta}^{\diamond}(\Omega)} \frac{b_{\delta}\left(\boldsymbol{w}_{\delta}, q_{\delta}\right)}{\left\|\boldsymbol{w}_{\delta}\right\|_{V_{1 D}^{1}(\Omega) \times H_{1 D}^{1}(\Omega)}} \geq \beta_{\delta}\left\|q_{\delta}\right\|_{L_{1}^{2}(\Omega)},
$$


where

$$
\beta_{\delta}=c \bar{N}_{\delta}^{-\frac{1}{2}}\left(\log \bar{N}_{\delta}\right)^{-1} \quad \text { with } \quad \bar{N}_{\delta}=\max \left\{N_{\ell}, 1 \leq \ell \leq L\right\} .
$$

Proof. For already explained reasons, we only establish the second part of the lemma and prove it with $b_{\delta}(\cdot, \cdot)$ replaced by $b(\cdot, \cdot)$. Let $q_{\delta}$ belong to $M_{\delta(0)}$. We take

$$
q_{\delta}=\bar{q}_{\delta}+\tilde{q}_{\delta} \quad \text { with }\left.\quad \bar{q}_{\delta}\right|_{\Omega_{\ell}}=\frac{1}{\operatorname{meas}\left(\Omega_{\ell}\right)} \int_{\Omega_{\ell}} q_{\ell}(r, z) r d r d z .
$$

1) On each $\Omega_{\ell}$, we remark that $\tilde{q}_{\ell}=\left.\tilde{q}_{\delta}\right|_{\Omega_{\ell}}$ belongs to $\mathbb{P}_{N_{\ell}-2}\left(\Omega_{\ell}\right)$ and has a null weighted integral on $\Omega_{\ell}$. Let $\mathbb{P}_{N_{\ell}}^{0}\left(\Omega_{\ell}\right)$ stand for the subspace of $\mathbb{P}_{N}^{0}\left(\Omega_{\ell}\right)$ made of polynomials vahising on $\partial \Omega_{\ell}$. Thus, according to [2, Proposition X.2.5] and [6, Sections 24 and 25], there exists $\tilde{\boldsymbol{w}}_{\ell}$ in $\mathbb{P}_{N_{\ell}}^{0}\left(\Omega_{\ell}\right) \times \mathbb{P}_{N_{\ell}}^{0}\left(\Omega_{\ell}\right)$ such that $b\left(\tilde{\boldsymbol{w}}_{\ell}, \tilde{q}_{\ell}\right)=\left\|\tilde{q}_{\ell}\right\|_{L_{1}^{2}\left(\Omega_{\ell}\right)}^{2}$ and

$$
\left\|\tilde{\boldsymbol{w}}_{\delta}\right\|_{V_{1}^{1}\left(\Omega_{\ell}\right) \times H_{1}^{1}\left(\Omega_{\ell}\right)} \leq \tilde{c} \sqrt{N_{\ell}} \log N_{\ell}\left\|\tilde{q}_{\ell}\right\|_{L_{1}^{2}\left(\Omega_{\ell}\right)} .
$$

We take $\tilde{\boldsymbol{w}}_{\delta}$ such that $\left.\tilde{\boldsymbol{w}}_{\delta}\right|_{\Omega_{\ell}}=\tilde{\boldsymbol{w}}_{\ell}$. It is readily checked that $\tilde{\boldsymbol{w}}_{\delta}$ belongs to $\mathbb{X}_{\delta}^{0}(\Omega)^{2}$. Moreover we have

$$
b\left(\tilde{\boldsymbol{w}}_{\delta}, \tilde{q}_{\delta}\right)=\left\|\tilde{q}_{\delta}\right\|_{L_{1}^{2}(\Omega)}^{2} \quad \text { and } \quad\left\|\tilde{\boldsymbol{w}}_{\delta}\right\|_{V_{1 D}^{1}(\Omega) \times H_{1 D}^{1}(\Omega)} \leq \tilde{c} \bar{N}_{\delta}^{\frac{1}{2}} \log \bar{N}_{\delta}\left\|\tilde{q}_{\delta}\right\|_{L_{1}^{2}(\Omega)}
$$

2) Since $\bar{q}_{\delta}$ is constant on each subdomain $\Omega_{\ell}$ and according to [2, Lemma XI.1.1] (see also [2, Prop. XI.1.7]), there exists $\overline{\boldsymbol{w}}_{\delta}$ in $\mathbb{X}_{\delta}^{0}(\Omega) \times \mathbb{X}_{\delta}^{\diamond}(\Omega)$ such that

$$
b\left(\overline{\boldsymbol{w}}_{\delta}, \bar{q}_{\delta}\right)=\left\|\bar{q}_{\delta}\right\|_{L_{1}^{2}(\Omega)}^{2} \quad \text { and } \quad\left\|\overline{\boldsymbol{w}}_{\delta}\right\|_{V_{1 D}^{1}(\Omega) \times H_{1 D}^{1}(\Omega)} \leq \bar{c}\left\|\bar{q}_{\delta}\right\|_{L_{1}^{2}(\Omega)} .
$$

3) We now use the Boland and Nicolaides argument [9] and take : $\boldsymbol{w}_{\delta}=\tilde{\boldsymbol{w}}_{\delta}+\lambda \overline{\boldsymbol{w}}_{\delta}$ for a positive constant $\lambda$. Since $b\left(\tilde{\boldsymbol{w}}_{\delta}, \bar{q}_{\delta}\right)=0$, we have

$$
\begin{aligned}
b_{\delta}\left(\boldsymbol{w}_{\delta}, q_{\delta}\right)=\left\|\tilde{q}_{\delta}\right\|_{L_{1}^{2}(\Omega)}^{2}+\lambda\left\|\bar{q}_{\delta}\right\|_{L_{1}^{2}(\Omega)}^{2}-\bar{c} \lambda\left\|\tilde{q}_{\delta}\right\|_{L_{1}^{2}(\Omega)}\left\|\bar{q}_{\delta}\right\|_{L_{1}^{2}(\Omega)} & \\
& \geq\left(1-\frac{\bar{c}^{2} \eta^{2}}{2}\right)\left\|\tilde{q}_{\delta}\right\|_{L_{1}^{2}(\Omega)}^{2}+\lambda\left(1-\frac{\lambda}{2 \eta^{2}}\right)\left\|\bar{q}_{\delta}\right\|_{L_{1}^{2}(\Omega)}^{2},
\end{aligned}
$$

for any $\eta>0$. When taking $\eta=\frac{1}{\bar{c}}$ and $\lambda=\eta^{2}$ we deduce:

$$
b_{\delta}\left(\boldsymbol{w}_{\delta}, q_{\delta}\right) \geq \inf \left\{\frac{1}{2 \bar{c}^{2}}, \frac{1}{2}\right\}\left\|q_{\delta}\right\|_{L_{1}^{2}(\Omega)}^{2} .
$$

By using (3.11) and (3.12), we obtain

$$
\left\|\boldsymbol{w}_{\delta}\right\|_{\mathbb{Z}} \leq c \bar{N}_{\delta}^{\frac{1}{2}}\left(\log \bar{N}_{\delta}\right)\left\|q_{\delta}\right\|_{L_{1}^{2}(\Omega)} .
$$

Finally by combining (3.13) and (3.14) we obtain the inf-sup condition (3.9).

Even if condition (3.9) is not optimal, it is well-known that it cannot be improved, see [2, Prop. X.2.5] or [6, Thm 25.5]. In any case, it is sufficient for proving the next result. 
Proposition 3.3 If condition (3.8) holds, for any data $\left(f_{r}, f_{z}\right)$ and $\left(g_{r}, g_{z}\right)$ continuous on $\bar{\Omega}$ and satisfying the null flux condition (2.3), problem (3.7) has a unique solution $\left(u_{r, \delta}, u_{z, \delta}, p_{\delta}\right)$ in $\mathbb{X}_{\delta}^{*}(\Omega) \times \mathbb{X}_{\delta}(\Omega) \times \mathbb{M}_{\delta(0)}(\Omega)$. Moreover, in the case of homogeneous boundary conditions $g_{r}=g_{z}=0$, this solution satisfies, for a constant $c$ only depending on $\Omega$ and its decomposition (3.1),

$$
\left\|u_{r, \delta}\right\|_{V_{1 D}^{1}(\Omega)}+\left\|u_{z, \delta}\right\|_{H_{1 D}^{1}(\Omega)}+\beta_{\delta}\left\|p_{\delta}\right\|_{L_{1}^{2}(\Omega)} \leq c\left(\left\|\mathcal{I}_{\delta} f_{r}\right\|_{L_{1}^{2}(\Omega)}+\left\|\mathcal{I}_{\delta} f_{z}\right\|_{L_{1}^{2}(\Omega)}\right) .
$$

We prefer not to state the stability property in the general case since it is more complex.

\subsection{The discrete problems in the general case}

There also, the discrete problems are constructed by the Galerkin method with numerical integration. For all $k \neq 0$, they read

$$
\begin{aligned}
& \text { Find }\left(\boldsymbol{u}_{\delta}^{k}, p_{\delta}^{k}\right) \text { in } \mathbb{X}_{\delta(k)}(\Omega) \times \mathbb{M}_{\delta}(\Omega) \text {, with } \boldsymbol{u}_{\delta}^{k}-\mathcal{I}_{\delta} \boldsymbol{g}^{k} \text { in } \mathbb{Y}^{\diamond}(\Omega)^{3}, \text { such that } \\
& \qquad \begin{array}{c}
\forall \boldsymbol{v}_{\delta} \in \mathbb{X}_{\delta(k)}^{\diamond}(\Omega), \quad \mathcal{A}_{k, \delta}\left(\boldsymbol{u}_{\delta}^{k}, \boldsymbol{v}_{\delta}\right)+\mathcal{B}_{k, \delta}\left(\boldsymbol{v}_{\delta}, p_{\delta}^{k}\right)=\left(\boldsymbol{f}^{k}, \overline{\boldsymbol{v}}_{\delta}\right)_{\delta} \\
\forall q_{\delta} \in \mathbb{M}_{\delta}(\Omega), \quad \mathcal{B}_{k, \delta}\left(\boldsymbol{u}_{\delta}^{k}, q_{\delta}\right)=0
\end{array}
\end{aligned}
$$

where the sesquilinear forms $\mathcal{A}_{k, \delta}(\cdot, \cdot)$ and $\mathcal{B}_{k, \delta}(\cdot, \cdot)$ are now defined by

$$
\begin{aligned}
& \mathcal{A}_{k, \delta}\left(\boldsymbol{u}_{\delta}, \boldsymbol{w}_{\delta}\right)=a_{0 \delta}\left(u_{r, \delta}, w_{r, \delta}\right)+a_{0 \delta}\left(u_{\theta, \delta}, w_{\theta, \delta}\right)+a_{0 \delta}\left(u_{z, \delta}, w_{z, \delta}\right) \\
& \quad+\left(1+k^{2}\right)\left(r^{-1} u_{r, \delta}, r^{-1} \bar{w}_{r, \delta}\right)_{\delta}+\left(1+k^{2}\right)\left(r^{-1} u_{\theta, \delta}, r^{-1} \bar{w}_{\theta, \delta}\right)_{\delta} \\
& +2 i k\left(r^{-1} u_{\theta, \delta}, r^{-1} \bar{w}_{r, \delta}\right)_{\delta}-2 i k\left(r^{-1} u_{r, \delta}, r^{-1} \bar{w}_{\theta, \delta}\right)_{\delta}+k^{2}\left(r^{-1} u_{z, \delta}, r^{-1} \bar{w}_{z, \delta}\right)_{\delta}
\end{aligned}
$$

and

$$
\mathcal{B}_{k, \delta}\left(\boldsymbol{w}_{\delta}, q_{\delta}\right)=-\left(q_{\delta}, \partial_{r} \bar{w}_{r, \delta}+r^{-1}\left(\overline{w_{r, \delta}+i k w_{\theta, \delta}}\right)+\partial_{z} \bar{w}_{z, \delta}\right)_{\delta} .
$$

Despite the complex aspect of the forms $\mathcal{A}_{k, \delta}(\cdot, \cdot)$ and $\mathcal{B}_{k, \delta}(\cdot, \cdot)$, proving the well-posedness of the previous problem is simpler than for problem (3.7). Indeed, the continuity and ellipticity of $\mathcal{A}_{k, \delta}(\cdot, \cdot)$ immediately follows from the properties of the Gauss-Lobatto formulas, see [6, Remark 13.3] and [2, Lemma VI.1.4], due to the definition of the norm $\|\cdot\|_{\boldsymbol{H}_{(k)}^{1}(\Omega)}$ introduced in Section 2 (see also $[2$, Section X.1]). We hide here an obvious definition for the norm $\|\cdot\|_{\boldsymbol{H}_{(k) D}^{1}(\Omega)}$.

Lemma 3.4 The form $\mathcal{A}_{k, \delta}(\cdot, \cdot)$ is continuous on $\mathbb{X}_{\delta(k)}(\Omega) \times \mathbb{X}_{\delta(k)}(\Omega)$ and elliptic on $\mathbb{X}_{\delta(k)}^{\diamond}(\Omega)$, with norm and ellipticity constant independent of $\delta$.

The continuity of the form $\mathcal{B}_{k, \delta}(\cdot, \cdot)$ also follows from the properties of the Gauss-Lobatto formulas. Moreover, since no global or matching condition appears in the definition of $\mathbb{M}_{\delta}(\Omega)$, the inf-sup condition is easily derived from local ones. We refer to [2, Eq. X.2.32] for this. 
Lemma 3.5 The form $\mathcal{B}_{k, \delta}(\cdot, \cdot)$ is continuous on $\mathbb{X}_{\delta(k)}(\Omega) \times \mathbb{M}_{\delta}(\Omega)$, with norm independent of $\delta$. Moreover, for all $k \neq 0$ and for any $q_{\delta} \in \mathbb{M}_{\delta}(\Omega)$, there holds the inf-sup condition

$$
\sup _{\boldsymbol{w}_{\delta} \in \mathbb{X}_{\delta(k)}^{\diamond}(\Omega)} \frac{\mathcal{B}_{k, \delta}\left(\boldsymbol{w}_{\delta}, q_{\delta}\right)}{\left\|\boldsymbol{w}_{\delta}\right\|_{\boldsymbol{H}_{(k) D}^{1}}(\Omega)} \geq \beta_{\delta(k)}\left\|q_{\delta}\right\|_{L_{1}^{2}(\Omega)}
$$

where

$$
\beta_{\delta(k)}=c|k|^{-1} \bar{N}_{\delta}^{-\frac{1}{2}}\left(\log \bar{N}_{\delta}\right)^{-1},
$$

with $\bar{N}_{\delta}$ defined in (3.10).

All this leads to the well-posedness property.

Proposition 3.6 For all $k \neq 0$ and for any data $\boldsymbol{f}^{k}$ and $\boldsymbol{g}^{k}$ continuous on $\bar{\Omega}$, problem (3.16) has a unique solution $\left(\boldsymbol{u}_{\delta}^{k}, p_{\delta}^{k}\right)$ in $\mathbb{X}_{\delta(k)}(\Omega) \times \mathbb{M}_{\delta}(\Omega)$. Moreover, in the case of homogeneous boundary conditions $\boldsymbol{g}^{k}=\mathbf{0}$, this solution satisfies, for a constant $c$ only depending on $\Omega$ and its decomposition (3.1),

$$
\left\|\boldsymbol{u}_{\delta}^{k}\right\|_{\boldsymbol{H}_{(k) D}^{1}(\Omega)}+\beta_{\delta(k)}\left\|p_{\delta}^{k}\right\|_{L_{1}^{2}(\Omega)} \leq c\left\|\mathcal{I}_{\delta} \boldsymbol{f}^{k}\right\|_{L_{1}^{2}(\Omega)^{3}} .
$$




\section{Error estimates}

We prove the a priori error estimates concerning first the solution of problem (3.7), second the solution of problem (3.16). We conclude with an estimate on the whole domain $\breve{\Omega}$.

\subsection{The case of axisymmetric data}

For simplicity, we denote by $\mathbb{Z}_{\delta}$ the product $\mathbb{X}_{\delta}^{*}(\Omega) \times \mathbb{X}_{\delta}(\Omega)$ and by $\mathbb{Z}_{\delta}^{\diamond}$ the product $\mathbb{X}_{\delta}^{0}(\Omega) \times \mathbb{X}_{\delta}^{\diamond}(\Omega)$, by $\|\cdot\|_{\boldsymbol{Z}}$ the norm on the product space $V_{1 D}^{1}(\Omega) \times H_{1 D}^{1}(\Omega)$. We define the space $\mathbb{V}_{\delta}$ by :

$$
\mathbb{V}_{\delta}=\left\{\boldsymbol{w}_{\delta} \in \mathbb{Z}_{\delta}^{\diamond} ; \forall q_{\delta} \in \mathbb{M}_{\delta}(\Omega), b_{\delta}\left(\boldsymbol{w}_{\delta}, q_{\delta}\right)=0\right\}
$$

In the case of homogeneous boundary data $g_{r}=g_{z}=0$, to prove an estimate between the solutions $\boldsymbol{u}$ of problem (2.6) and $\boldsymbol{u}_{\delta}$ of problem (3.7), we first use the Strang Lemma: With obvious notation for the bilinear form $\mathcal{A}$,

$$
\begin{aligned}
\left\|\boldsymbol{u}-\boldsymbol{u}_{\delta}\right\|_{\boldsymbol{Z}} \leq c\left(\inf _{\boldsymbol{v}_{\delta} \in \mathbb{V}_{\delta}}\right. & \left\|\boldsymbol{u}-\boldsymbol{v}_{\delta}\right\|_{\boldsymbol{Z}}+\inf _{q_{\delta} \in \mathbb{M}_{\delta}(\Omega)}\left\|p-q_{\delta}\right\|_{L_{1}^{2}(\Omega)} \\
+\sup _{\boldsymbol{w}_{\delta} \in \mathbb{Z}_{\delta}} \frac{\mathcal{A}\left(\boldsymbol{v}_{\delta}, \boldsymbol{w}_{\delta}\right)-\mathcal{A}_{\delta}\left(\boldsymbol{v}_{\delta}, \boldsymbol{w}_{\delta}\right)}{\left\|\boldsymbol{w}_{\delta}\right\|_{\boldsymbol{Z}}} & \\
+\sup _{\boldsymbol{z}_{\delta} \in \mathbb{Z}_{\delta}} \frac{\int_{\Omega} \boldsymbol{f}(r, z) \cdot \boldsymbol{z}_{\delta}(r, z) r d r d z-\left(\mathcal{I}_{\delta} \boldsymbol{f}, \boldsymbol{z}_{\delta}\right)_{\delta}}{\left\|\boldsymbol{z}_{\delta}\right\|_{\boldsymbol{Z}}} & \left.+\sup _{\boldsymbol{y}_{\delta} \in \mathbb{Z}_{\delta}} \frac{\sum_{\mu=1}^{M^{-}} \int_{\gamma_{\mu}^{-}}\left(\frac{\partial \boldsymbol{u}}{\partial \boldsymbol{n}_{\mu}}+p \boldsymbol{n}\right)(\tau) \cdot\left[\boldsymbol{y}_{\delta}\right](\tau) d \tau}{\left\|\boldsymbol{y}_{\delta}\right\|_{\boldsymbol{Z}}}\right),
\end{aligned}
$$

where $c$ is a positive constant independent of $\delta$.

Even if the previous estimate seems rather complex, it can be noted that the terms due to numerical integration, i.e. on the second and third lines, can be evaluated separately on each $\Omega_{\ell}$. So bounding them relies on the exactness properties of the quadrature formula and standard approximation and interpolation results [2, Sections 5.2 and 6.3]. Similarly, due to the definition of $\mathbb{M}_{\delta}(\Omega)$, the same arguments yield, for any $s_{\ell} \geq 0$,

$$
\inf _{q_{\delta} \in \mathbb{M}_{\delta}(\Omega)}\left\|p-q_{\delta}\right\|_{L_{1}^{2}(\Omega)} \leq c \sum_{\ell=1}^{L} N_{\ell}^{-s_{\ell}}\|p\|_{H_{1}^{s_{\ell}}\left(\Omega_{\ell}\right)} .
$$

So it remains to bound the approximation error $\inf _{\boldsymbol{v}_{\delta} \in \mathbb{V}_{\delta}}\left\|\boldsymbol{u}-\boldsymbol{v}_{\delta}\right\|_{\boldsymbol{Z}}$ and the consistency term in the fourth line of Strang's inequality. 
Proposition 4.1 Assume that the part $\boldsymbol{u}=\left(u_{r}, u_{z}\right)$ of the solution of problem (2.6) with homogeneous boundary conditions is such that each $\boldsymbol{u}_{\mid \Omega_{\ell}}$ belongs to $H_{1}^{s_{\ell}+1}\left(\Omega_{\ell}\right)^{2}$, with $s_{\ell}>\frac{3}{2}$ for $1 \leq \ell \leq L_{0}$ and $s_{\ell}>\frac{1}{2}$ otherwise. If condition (3.8) holds, there exists a function $\boldsymbol{v}_{\delta}$ in $\mathbb{V}_{\delta}$ such that:

$$
\left\|\boldsymbol{u}-\boldsymbol{v}_{\delta}\right\|_{\boldsymbol{Z}} \leq c \lambda_{\delta}^{\frac{3}{4}} \sum_{\ell=1}^{L} N_{\ell}^{-s_{\ell}}\|\boldsymbol{u}\|_{H_{1}^{s_{\ell}+1}\left(\Omega_{\ell}\right)^{2}} .
$$

where

$$
\lambda_{\delta}=\max \left\{\frac{N_{\mu}^{+}}{N_{m}^{-}}, \frac{N_{m}^{-}}{N_{\mu}^{+}}\right\}
$$

the maximum being taken on all mortars $\gamma_{\mu}^{+}, 1 \leq \mu \leq M^{+}$, and non-mortars $\gamma_{m}^{-}, 1 \leq m \leq M^{-}$, such that $\gamma_{\mu}^{+} \cap \gamma_{m}^{-}$has a positive measure.

Proof. Since it is very complex, we only give an abridged version and refer to [11, Prop. 3.2.3] for details (see also [3] for similar arguments). The function $\boldsymbol{v}_{\delta}$ is built as the sum $\boldsymbol{v}_{\delta}^{1}+\boldsymbol{v}_{\delta}^{2}+\boldsymbol{v}_{\delta}^{3}$.

1) Construction of $\boldsymbol{v}_{\delta}^{1}$ : Since $\boldsymbol{u}$ is divergence-free in the sense

$$
\partial_{r} u_{r, \delta}+r^{-1} u_{r, \delta}+\partial_{z} u_{z, \delta}=0 \quad \text { on } \Omega,
$$

there exists a function $\psi$ in $H_{1}^{2}(\Omega)$ such that

$$
\boldsymbol{u}=\boldsymbol{R o t}_{a}(\psi)=\left(\partial_{z} \psi,-\frac{1}{r} \partial_{r}(r \psi)\right)
$$

Setting $\psi_{\ell}=\left.\psi\right|_{\Omega_{\ell}}$, the idea is to take $\boldsymbol{v}_{\delta}^{1}$ such that $\left.\boldsymbol{v}_{\delta}^{1}\right|_{\Omega_{\ell}}=\boldsymbol{R o t}_{a}\left(\tilde{\Pi}_{N_{\ell}}^{*, 2} \psi_{\ell}\right)$, where each $\tilde{\Pi}_{N_{\ell}}^{*, 2}$ is an appropriate projection operator from $H_{1}^{2}\left(\Omega_{\ell}\right)$ onto $\mathbb{P}_{N_{\ell}}\left(\Omega_{\ell}\right)$ preserving the nullity of the function and its normal derivative on the edges of $\Omega_{\ell}$, and also the values at the corners of the $\Omega_{\ell}$. Then, the function $\boldsymbol{v}_{\delta}^{1}$ is still divergence-free on each $\Omega_{\ell}$.

2) Construction of $\boldsymbol{v}_{\delta}^{2}$ : For $1 \leq \mu \leq M^{+}$, let $a_{p}^{\mu}, 1 \leq p \leq P_{\mu}$, be the corners of the $\Omega_{\ell}$ which are inside $\gamma_{\mu}^{+}$. With each of them, we associate a tensorized polynomial $\eta_{p}$ in $\mathbb{P}_{N_{\mu}^{+}}\left(\Omega_{\mu}^{+}\right)$which vanishes on $\partial \Omega_{\mu}^{+} \backslash \gamma_{\mu}^{+}$and moreover satisfies

$$
\eta_{p}\left(a_{p}\right)=1 \quad \text { and } \quad \eta_{p}\left(a_{p^{\prime}}\right)=0, \quad 1 \leq p^{\prime} \leq P_{\mu}, p^{\prime} \neq p .
$$

Note that this requires condition (3.8). Thus, we set

$$
\eta_{\mu, p}^{*}= \begin{cases}\eta_{p} & \text { in } \Omega_{\mu}^{+}, \\ 0 & \text { in } \Omega \backslash \bar{\Omega}_{\mu}^{+} .\end{cases}
$$

The idea is to take, with obvious notation,

$$
z_{\delta}=\sum_{\mu=1}^{M^{+}} \sum_{p=1}^{P_{\mu}}\left(\psi-\tilde{\Pi}_{\delta}^{*, 2} \psi\right)\left(a_{p}\right) \eta_{\mu, p}^{*},\left.\quad v_{\delta}^{2}\right|_{\Omega_{\ell}}=\boldsymbol{R o t}_{a}\left(\left.z_{\delta}\right|_{\Omega_{\ell}}\right) .
$$


3) Construction of $\boldsymbol{v}_{\delta}^{3}$ : Setting $\left.z_{\delta}^{12}\right|_{\Omega_{\ell}}=\tilde{\Pi}_{N_{\ell}}^{*, 2} \psi_{\ell}+\left.z_{\delta}\right|_{\Omega_{\ell}}$, we define

$$
\sigma_{\gamma_{m}^{-}}=\tilde{\pi}_{N_{m}^{-}-2}^{*, 2}\left(\left[z_{\delta}^{12}\right]_{\gamma_{m}^{-}}\right), \quad \sigma_{\gamma_{m}^{-}}^{n}=\partial_{n}\left(\tilde{\pi}_{N_{m}^{-}-2}^{* 2}\left(\left[z_{\delta}^{12}\right]_{\gamma_{m}^{-}}\right),\right.
$$

where $[\cdot]_{\gamma_{m}^{-}}$stands for the jump through $\gamma_{m}^{-}$(with the right sign) and the operator $\tilde{\pi}_{N_{m}^{-}-2}^{*, 2}$ is an appropriate projection operator onto $\mathbb{P}_{N_{m}^{-}-2}\left(\gamma_{m}^{-}\right)$. Next, on each $\gamma_{m}^{-}$, we use a lifting operator $\mathcal{R}^{2, \gamma_{m}^{-}}$of the trace and normal derivative and set

$$
\boldsymbol{v}_{\delta}^{3}=\sum_{m=1}^{M^{-}} \boldsymbol{R o t}_{a} \circ \mathcal{R}^{2, \gamma_{m}^{-}}\left(\sigma_{\gamma_{m}^{-}}, \sigma_{\gamma_{m}^{-}}^{n}\right) .
$$

The function $\boldsymbol{v}_{\delta}=\boldsymbol{v}_{\delta}^{1}+\boldsymbol{v}_{\delta}^{2}+\boldsymbol{v}_{\delta}^{3}$ now belongs to $\mathbb{V}_{\delta}$. Estimate (4.2) is proved in [11, Prop. 3.2.3].

We say that the decomposition is conforming if the intersection of two different domains $\bar{\Omega}_{\ell}$ is either empty or a common vertex or a whole edge of both of them. The result of Proposition 4.1 can be improved in this case, since the step 2 of its proof, i.e. the construction of $\boldsymbol{v}_{\delta}^{2}$, can be omitted.

Corollary 4.2 Assume that the part $\boldsymbol{u}=\left(u_{r}, u_{z}\right)$ of the solution of problem (2.6) with homogenous boundary conditions is such that each $\boldsymbol{u}_{\mid \Omega_{\ell}}$ belongs to $H_{1}^{s_{\ell}+1}\left(\Omega_{\ell}\right)$, with $s_{\ell}>\frac{3}{2}$ for $1 \leq \ell \leq L_{0}$ and $s_{\ell}>\frac{1}{2}$ otherwise. In the case of a conforming decomposition, there exists a function $\boldsymbol{v}_{\delta}$ in $\mathbb{V}_{\delta}$ such that:

$$
\left\|\boldsymbol{u}-\boldsymbol{v}_{\delta}\right\|_{\boldsymbol{Z}} \leq c \sum_{\ell=1}^{L} N_{\ell}^{-s_{\ell}}\left\|\boldsymbol{u}_{\ell}\right\|_{H_{1}^{s_{\ell}+1}\left(\Omega_{\ell}\right)^{2}} .
$$

Unfortunately, the previous estimates do not extend to the case of nonhomogeneous boundary conditions, and we are led to use the formula (see [10, Chap. II, Eq. (1.16)] for instance) in this case:

$$
\inf _{\boldsymbol{v}_{\delta} \in \mathbb{V}_{\delta}}\left\|\boldsymbol{w}-\boldsymbol{v}_{\delta}\right\|_{\boldsymbol{Z}} \leq \beta_{\delta}^{-1} \inf _{\boldsymbol{z}_{\delta} \in \mathbb{Z}_{\delta}^{\diamond}}\left\|\boldsymbol{w}-\boldsymbol{z}_{\delta}\right\|_{\boldsymbol{Z}}
$$

Since $\beta_{\delta}$ is not bounded independently of $\delta$, see (3.10), this leads to a lack of optimality. We now treat the consistency error.

Proposition 4.3 For any function $\varphi$ such that each $\left.\varphi\right|_{\Omega_{\ell}}, 1 \leq \ell \leq L$, belongs to $H_{1}^{s_{\ell}}\left(\Omega_{\ell}\right)$, with $s_{\ell}>1$ for $1 \leq \ell \leq L_{0}$ and $s_{\ell}>0$ otherwise, the following estimate holds for all $w_{\delta}$ in $\mathbb{X}_{\delta}$

$$
\left|\sum_{\gamma_{m}^{-} \in \mathcal{S}} \int_{\gamma_{m}^{-}} \varphi(\tau)\left[w_{\delta}\right](\tau) d \tau\right| \leq c\left(\sum_{\ell=1}^{L} N_{\ell}^{-s_{\ell}}\left(\log N_{\ell}\right)^{\varrho \ell}\|\varphi\|_{H_{1}^{s_{\ell}\left(\Omega_{\ell}\right)}}\right)\left\|w_{\delta}\right\|_{H_{1 D}^{1}(\Omega)}
$$

where $\varrho_{\ell}$ is equal to 1 if one of the sides of $\Omega_{\ell}$ is a $\gamma_{m}^{-}$and intersects at least two subdomains $\bar{\Omega}_{\ell^{\prime}}, \ell^{\prime} \neq \ell$, and 0 otherwise. 
Proof. It follows from the definition (3.2) of the space $\mathbb{X}_{\delta}(\Omega)$ that, for any $\psi$ in $\mathbb{P}_{N_{m}^{-}-2}\left(\gamma_{m}^{-}\right)$,

$$
\int_{\gamma_{\bar{m}}^{-}} \varphi(\tau)\left[w_{\delta}\right](\tau) d \tau=\int_{\gamma_{\bar{m}}}(\varphi-\psi)(\tau)\left[w_{\delta}\right](\tau) d \tau
$$

whence, for an appropriate space $T\left(\gamma_{m}^{-}\right)$,

$$
\left|\int_{\gamma_{m}^{-}} \varphi(\tau)\left[w_{\delta}\right](\tau) d \tau\right| \leq\|\varphi-\psi\|_{T\left(\gamma_{m}^{-}\right)^{\prime}}\left\|\left[w_{\delta}\right]\right\|_{T\left(\gamma_{m}^{-}\right)}
$$

Next, it follows from [5, Chap. I, Thm 8.3] that the trace operator maps $H_{1}^{1}\left(\Omega_{m}^{-}\right)$ onto $H^{\frac{1}{2}}\left(\gamma_{m}^{-}\right)$or $H_{1}^{\frac{1}{2}}\left(\gamma_{m}^{-}\right)$according as $\gamma_{m}^{-}$is parallel to the axis $(O z)$ or $(O r)$. Similarly, it maps $H_{1}^{1}\left(\Omega_{\ell}\right)$ onto $H^{\frac{1}{2}}\left(\gamma_{m}^{-}\right)$or $H_{1}^{\frac{1}{2}}\left(\gamma_{m}^{-}\right)$if $\gamma_{m}^{-}$is contained in an edge of $\Omega_{\ell}$ and the product of the spaces $H_{1}^{1}\left(\Omega_{\ell_{i}}\right)$ onto $H^{\frac{1}{2}-\varepsilon}\left(\gamma_{m}^{-}\right)$or $H_{1}^{\frac{1}{2}-\varepsilon}\left(\gamma_{m}^{-}\right)$with norm $\leq c \varepsilon^{-1}$ (see [8]) if $\gamma_{m}^{-}$is contained in the union of edges of the $\Omega_{\ell_{i}}$. The desired estimate follows by taking $T\left(\gamma_{m}^{-}\right)$equal to this trace space, choosing $\psi$ equal to the image of $\varphi$ by the orthogonal projection operator from $L^{2}\left(\gamma_{m}^{-}\right)$or $L_{1}^{2}\left(\gamma_{m}^{-}\right)$onto $\mathbb{P}_{N_{m}^{-}-2}\left(\gamma_{m}^{-}\right)$and using standard duality arguments, finally taking $\varepsilon=\left(\log \left(N_{m}^{-}\right)\right)^{-1}$.

From Propositions 4.1 and 4.3 combined with the previous arguments, we easily derive the estimate for $\left\|\boldsymbol{u}-\boldsymbol{u}_{\delta}\right\|_{\boldsymbol{z}}$. Thus, the estimate on the pressure follows from the inf-sup condition (3.9), combined with the previous result. All this leads to the next statement. From now on, we assume for simplicity that condition (3.8) holds.

Theorem 4.4 Let $(\boldsymbol{u}, p)$ be the solution of problem (2.6) such that each $\left.(\boldsymbol{u}, p)\right|_{\Omega_{\ell}}$, $1 \leq \ell \leq L$, belongs to $H_{1}^{s_{\ell}+1}\left(\Omega_{\ell}\right)^{2} \times H_{1}^{s_{\ell}}\left(\Omega_{\ell}\right)$, with $s_{\ell}>1$ for $1 \leq \ell \leq L_{0}$ and $s_{\ell}>0$ otherwise. If moreover the data $\boldsymbol{f}$ are such that each $\left.\boldsymbol{f}\right|_{\Omega_{\ell}}, 1 \leq \ell \leq L$, belongs to $H_{1}^{\sigma_{\ell}}\left(\Omega_{\ell}\right)^{2}$, with $\sigma_{\ell}>\frac{3}{2}$ for $1 \leq \ell \leq L_{0}$ and $\sigma_{\ell}>1$ otherwise, the following error estimate holds between this solution and the solution $\left(\boldsymbol{u}_{\delta}, p_{\delta}\right)$ of problem (3.7):

(i) In the case of homogeneous boundary conditions,

$$
\begin{aligned}
\left\|\boldsymbol{u}-\boldsymbol{u}_{\delta}\right\|_{\boldsymbol{Z}}+\beta_{\delta} \| p & -p_{\delta} \|_{L_{1}^{2}(\Omega)} \\
\leq c & \left(\sum_{\ell=1}^{L}\left(1+\lambda_{\ell}\right)^{\frac{3}{4}} N_{\ell}^{-s_{\ell}}\left(\log N_{\ell}\right)^{\varrho_{\ell}}\|\boldsymbol{u}\|_{H_{1}^{s_{\ell}+1}\left(\Omega_{\ell}\right)^{2}}\right. \\
& \left.\quad+\sum_{\ell=1}^{L} N_{\ell}^{-s_{\ell}}\left(\log N_{\ell}\right)^{\varrho_{\ell}}\|p\|_{H_{1}^{s_{\ell}\left(\Omega_{\ell}\right)}}+\sum_{\ell=1}^{L} N_{\ell}^{-\sigma_{\ell}}\|\boldsymbol{f}\|_{H_{1}^{\sigma_{\ell}}\left(\Omega_{\ell}\right)^{2}}\right) .
\end{aligned}
$$


(ii) In the general case,

$$
\begin{aligned}
\left\|\boldsymbol{u}-\boldsymbol{u}_{\delta}\right\|_{\boldsymbol{Z}}+ & \beta_{\delta}\left\|p-p_{\delta}\right\|_{L_{1}^{2}(\Omega)} \\
\leq c\left(\beta_{\delta}^{-1} \sum_{\ell=1}^{L}\left(1+\lambda_{\ell}\right)^{\frac{1}{2}} N_{\ell}^{-s_{\ell}}\left(\log N_{\ell}\right)^{\varrho \ell}\|\boldsymbol{u}\|_{H_{1}^{s_{\ell}+1}\left(\Omega_{\ell}\right)^{2}}\right. & \\
& \left.\quad+\sum_{\ell=1}^{L} N_{\ell}^{-s_{\ell}}\left(\log N_{\ell}\right)^{\varrho_{\ell}}\|p\|_{H_{1}^{s_{\ell}}\left(\Omega_{\ell}\right)}+\sum_{\ell=1}^{L} N_{\ell}^{-\sigma_{\ell}}\|\boldsymbol{f}\|_{H_{1}^{\sigma_{\ell}}\left(\Omega_{\ell}\right)^{2}}\right),
\end{aligned}
$$

where $\lambda_{\ell}$ and $\varrho_{\ell}$ are defined in Propositions 4.1 and 4.3, respectively.

These estimates are simpler and sometimes fully optimal when the decomposition is conforming.

Corollary 4.5 If the assumptions of Theorem 4.4 hold and in the case of a conforming decomposition, the following error estimate holds between the solutions $(\boldsymbol{u}, p)$ problem $(2.6)$ and $\left(\boldsymbol{u}_{\delta}, p_{\delta}\right)$ of problem (3.7):

(i) In the case of homogeneous boundary conditions,

$$
\begin{aligned}
& \left\|\boldsymbol{u}-\boldsymbol{u}_{\delta}\right\|_{\boldsymbol{Z}}+\beta_{\delta}\left\|p-p_{\delta}\right\|_{L_{1}^{2}(\Omega)} \\
& \quad \leq c\left(\sum_{\ell=1}^{L} N_{\ell}^{-s_{\ell}}\left(\|\boldsymbol{u}\|_{H_{1}^{s_{\ell}+1}\left(\Omega_{\ell}\right)^{2}}+\|p\|_{H_{1}^{s_{\ell}\left(\Omega_{\ell}\right)}}\right)+\sum_{\ell=1}^{L} N_{\ell}^{-\sigma_{\ell}}\|\boldsymbol{f}\|_{H_{1}^{\sigma_{\ell}}\left(\Omega_{\ell}\right)^{2}}\right) .
\end{aligned}
$$

(ii) In the general case,

$$
\begin{aligned}
& \left\|\boldsymbol{u}-\boldsymbol{u}_{\delta}\right\|_{\boldsymbol{Z}}+\beta_{\delta}\left\|p-p_{\delta}\right\|_{L_{1}^{2}(\Omega)} \\
\leq & c\left(\beta_{\delta}^{-1} \sum_{\ell=1}^{L} N_{\ell}^{-s_{\ell}}\|\boldsymbol{u}\|_{H_{1}^{s_{\ell}+1}\left(\Omega_{\ell}\right)^{2}}+\sum_{\ell=1}^{L} N_{\ell}^{-s_{\ell}}\|p\|_{H_{1}^{s_{\ell}\left(\Omega_{\ell}\right)}}+\sum_{\ell=1}^{L} N_{\ell}^{-\sigma_{\ell}}\|\boldsymbol{f}\|_{H_{1}^{\sigma_{\ell}\left(\Omega_{\ell}\right)^{2}}}\right) .
\end{aligned}
$$

To go further, we now give a more explicit estimate of the error, where the singularities of the solution are taken into account. We first recall that, since all the angles of $\Omega$ in the corners $c_{i}$ which belong to $\Gamma_{0}$ are equal to $\frac{\pi}{2}$, these corners do not give birth to any singular function. On the other hand, the angles $\omega_{e_{i}}$ of $\Omega$ in the corners $e_{i}$ are equal to $\frac{\pi}{2}$ or $\frac{3 \pi}{2}$. In a neighbourhood of this corner, the solution admits the expansion, where the singular functions $S_{\cdot, e_{i}}^{(n)}$ are defined in [2, Section IX.1.b],

$$
\begin{array}{r}
u_{r}=u_{r, r e g}+\beta_{u} S_{r, e_{i}}^{(0)}+\sum_{n \geq 1} \alpha_{u} S_{r, e_{i}}^{(n)}, \quad u_{z}=u_{z, r e g}+\beta_{u} S_{z, e_{i}}^{(0)}+\sum_{n \geq 1} \alpha_{u} S_{z, e_{i}}^{(n)}, \\
p=p_{\text {reg }}+\beta_{p} S_{p, e_{i}}^{(0)}+\sum_{n \geq 1} \alpha_{p} S_{p, e_{i}}^{(n)} .
\end{array}
$$


Moreover, the support of the singular functions is the union of the $\bar{\Omega}_{\ell}$ such that $c_{i}$ is a vertex of $\Omega_{\ell}$, their definition involves the positive quantities $\eta\left(\omega_{e_{i}}\right)$ (see $[2$, Section IX.1.b] for an explicit definition of the function $\eta$ ) and the approximation properties of these functions are well-known (we refer to [11, Section 3.2.4] for more details). We only consider the case of a conforming decomposition for simplicity and we denote by $N_{\delta}$ the minimum of the $N_{\ell}, 1 \leq \ell \leq L$.

Corollary 4.6 If the data $(\boldsymbol{f}, \boldsymbol{g})$ belong to $H_{1}^{s-1}(\Omega)^{2} \times H_{1}^{s+1}(\Omega)^{2}$ with $s>\frac{5}{2}$, the following error estimate holds between the solutions $(\boldsymbol{u}, p)$ of problem (2.6) and $\left(\boldsymbol{u}_{\delta}, p_{\delta}\right)$ of problem (3.7):

(i) In the case of homogeneous boundary conditions,

$$
\left\|\boldsymbol{u}-\boldsymbol{u}_{\delta}\right\|_{\boldsymbol{Z}}+\beta_{\delta}\left\|p-p_{\delta}\right\|_{L_{1}^{2}(\Omega)} \leq c\left(1+\lambda_{\delta}\right)^{\frac{3}{4}} \sup \left\{N_{\delta}^{1-s}, E_{\delta}^{S}\right\}\|\boldsymbol{f}\|_{H_{1}^{s-1}(\Omega)^{2}} .
$$

(ii) In the general case,

$$
\begin{aligned}
& \left\|\boldsymbol{u}-\boldsymbol{u}_{\delta}\right\|_{\boldsymbol{Z}}+\beta_{\delta}\left\|p-p_{\delta}\right\|_{L_{1}^{2}(\Omega)} \\
& \quad \leq c\left(1+\lambda_{\delta}\right)^{\frac{1}{2}} \sup \left\{N_{\delta}^{1-s}, \beta_{\delta}^{-1} E_{\delta}^{S}\right\}\left(\|\boldsymbol{f}\|_{H_{1}^{s-1}(\Omega)^{2}}+\|\boldsymbol{g}\|_{H_{1}^{s+1}(\Omega)^{2}}\right) .
\end{aligned}
$$

where

$$
\begin{aligned}
E_{\delta}^{S}=\max \left\{E_{\ell}^{S}, 1 \leq \ell \leq L\right\}, \\
E_{\ell}^{S}= \begin{cases}0 & \text { if } \bar{\Omega}_{\ell} \text { does not contain any } e_{i}, \\
N_{e_{i}}^{-2 \eta\left(\frac{\pi}{2}\right)}\left(\log N_{e_{i}}\right)^{\frac{1}{2}} & \text { if } \bar{\Omega}_{\ell} \text { contains } e_{i} \text { with } \omega_{e_{i}}=\frac{\pi}{2}, \\
N_{e_{i}}^{-2 \eta\left(\frac{3 \pi}{2}\right)}\left(\log N_{e_{i}}\right)^{\frac{1}{2}} & \text { if } \bar{\Omega}_{\ell} \text { contains } e_{i} \text { with } \omega_{e_{i}}=\frac{3 \pi}{2},\end{cases}
\end{aligned}
$$

and $N_{e_{i}}$ is the minimum of the $N_{\ell}$ for the $\Omega_{\ell}$ such that $c_{i}$ is a vertex of $\Omega_{\ell}$.

To make these last estimates fully complete, we recall [2, Section IX.1.b] that $\eta\left(\frac{\pi}{2}\right) \simeq 2,73959$ and $\eta\left(\frac{3 \pi}{2}\right) \simeq 0,54448$.

\subsection{The general case}

Owing to Lemmas 3.4 and 3.5, the same arguments as in Section 4.1 lead to similar estimates. However, for the sake of brevity, we prefer to state only the final result. We recall from [2, Section IX.1.b] that the singular functions exhibited above are the same for all values of $k$. We also introduce for any $s \geq 0$ the norm

$$
\|\boldsymbol{v}\|_{H_{(k)}^{s}(\Omega)}=\left|\boldsymbol{v} e^{i k \theta}\right|_{H^{s}(\breve{\Omega})}
$$

(note that the two definitions of $\|\cdot\|_{H_{(k)}^{1}(\Omega)}$ coincide) and by $\boldsymbol{H}_{(k)}^{s}(\Omega)$ the set of functions $\boldsymbol{v}$ in $L_{1}^{2}(\Omega)^{3}$ such that $\|\boldsymbol{v}\|_{H_{(k)}^{s}(\Omega)}<+\infty$. 
Theorem 4.7 If the data $\left(\boldsymbol{f}^{k}, \boldsymbol{g}^{k}\right)$ belong to $H_{(k)}^{s-1}(\Omega)^{3} \times H_{(k)}^{s+1}\left(\Omega_{\ell}\right)^{3}$ with $s>\frac{5}{2}$, the following error estimate holds between the solutions $\left(\boldsymbol{u}^{k}, p^{k}\right)$ of problem (2.2) and $\left(\boldsymbol{u}_{\delta}^{k}, p_{\delta}^{k}\right)$ of problem (3.16):

(i) In the case of homogeneous boundary conditions,

$\left\|\boldsymbol{u}^{k}-\boldsymbol{u}_{\delta}^{k}\right\|_{\boldsymbol{H}_{(k)}^{1}(\Omega)}+\beta_{\delta(k)}\left\|p^{k}-p_{\delta}^{k}\right\|_{L_{1}^{2}(\Omega)} \leq c\left(1+\lambda_{\delta}\right)^{\frac{3}{4}} \sup \left\{N_{\delta}^{1-s}, E_{\delta}^{S}\right\}\left\|\boldsymbol{f}^{k}\right\|_{\boldsymbol{H}_{(k)}^{s-1}(\Omega)^{2}}$.

(ii) In the general case,

$$
\begin{aligned}
& \left\|\boldsymbol{u}^{k}-\boldsymbol{u}_{\delta}^{k}\right\|_{\boldsymbol{H}_{(k)}^{1}(\Omega)}+\beta_{\delta(k)}\left\|p^{k}-p_{\delta}^{k}\right\|_{L_{1}^{2}(\Omega)} \\
& \quad \leq c\left(1+\lambda_{\delta}\right)^{\frac{1}{2}} \sup \left\{N_{\delta}^{1-s}, \beta_{\delta(k)}^{-1} E_{\delta}^{S}\right\}\left(\left\|\boldsymbol{f}^{k}\right\|_{\boldsymbol{H}_{(k)}^{s-1}(\Omega)}+\left\|\boldsymbol{g}^{k}\right\|_{\boldsymbol{H}_{(k)}^{s+1}(\Omega)}\right),
\end{aligned}
$$

where the quantities $\lambda_{\delta}$ and $E_{\delta}^{S}$ are introduced in Proposition 4.1 and Corollary 4.6 , respectively.

\subsection{Back to the three-dimensional problem}

Once the discrete coefficients $\left(\boldsymbol{u}_{\delta}^{k}, p_{\delta}^{k}\right),|k| \leq K$, are known, the basic idea is to define the three-dimensional discrete solution

$$
\begin{aligned}
\breve{\boldsymbol{u}}_{K, \delta}(r, \theta, z)=\frac{1}{\sqrt{2 \pi}} \sum_{|k| \leq K} \boldsymbol{u}_{\delta}^{k}(r, z) e^{i k \theta} & \\
\breve{p}_{K, \delta}(r, \theta, z) & =\frac{1}{\sqrt{2 \pi}} \sum_{|k| \leq K} p_{\delta}^{k}(r, z) e^{i k \theta} .
\end{aligned}
$$

Indeed, bounding the error between the solution $(\breve{\boldsymbol{u}}, \breve{p})$ of problem (1.1) and this solution relies on the triangle inequality (with obvious definition for the $\|\cdot\|_{\left.H_{D}^{1}(\breve{\Omega})^{-n o r m}\right)}$

$$
\left\|\breve{\boldsymbol{u}}-\breve{\boldsymbol{u}}_{K, \delta}\right\|_{H_{D}^{1}(\breve{\Omega})^{3}} \leq\left\|\breve{\boldsymbol{u}}-\breve{\boldsymbol{u}}_{K}\right\|_{H^{1}(\breve{\Omega})^{3}}+\left\|\breve{\boldsymbol{u}}_{K}-\breve{\boldsymbol{u}}_{K, \delta}\right\|_{H_{D}^{1}(\breve{\Omega})^{3}},
$$

and its analogue for $\left\|\breve{p}-\breve{p}_{K, \delta}\right\|_{L^{2}(\breve{\Omega})}$. The first term in the right-hand side of this inequality is evaluated in Proposition 2.4, while the second one obviously satisfies

$$
\left\|\breve{\boldsymbol{u}}_{K}-\breve{\boldsymbol{u}}_{K, \delta}\right\|_{H_{D}^{1}(\breve{\Omega})^{3}}^{2}=\sum_{|k| \leq K}\left\|\boldsymbol{u}^{k}-\boldsymbol{u}_{\delta}^{k}\right\|_{\boldsymbol{H}_{(k) D}^{1}(\Omega)}^{2} .
$$

So the final result is easily derived from Thoerem 4.7.

Theorem 4.8 Assume that the discretization parameters $K$ and $\delta$ satisfy

$$
K \leq N_{\delta}
$$


If the data $(\breve{\boldsymbol{f}}, \breve{\boldsymbol{g}})$ belong to $H^{s-1}(\breve{\Omega})^{2} \times H^{s+1}(\breve{\Omega})^{2}$ with $s>\frac{5}{2}$, the following error estimate holds between the solutions $(\breve{\boldsymbol{u}}, \breve{p})$ of problem $(1.1)$ and $\left(\breve{\boldsymbol{u}}_{K, \delta}, \breve{p}_{K, \delta}\right)$ defined in (4.8):

(i) In the case of homogeneous boundary conditions,

$$
\begin{aligned}
\left\|\breve{\boldsymbol{u}}-\breve{\boldsymbol{u}}_{K, \delta}\right\|_{H^{1}(\breve{\Omega})^{3}}+ & \beta_{\delta(K)}\left\|\breve{p}-\breve{p}_{K, \delta}\right\|_{L^{2}(\breve{\Omega})} \\
& \leq c\left(\left(1+\lambda_{\delta}\right)^{\frac{3}{4}} \sup \left\{N_{\delta}^{1-s}, E_{\delta}^{S}\right\}+K^{-s}\right)\|\breve{\boldsymbol{f}}\|_{H^{s-1}(\breve{\Omega})^{3}}
\end{aligned}
$$

(ii) In the general case,

$$
\begin{aligned}
&\left\|\breve{\boldsymbol{u}}-\breve{\boldsymbol{u}}_{K, \delta}\right\|_{H^{1}(\breve{\Omega})^{3}}+\beta_{\delta(K)}\left\|\breve{p}-\breve{p}_{K, \delta}\right\|_{L^{2}(\breve{\Omega})} \\
& \leq c\left(\left(1+\lambda_{\delta}\right)^{\frac{1}{2}} \sup \left\{N_{\delta}^{1-s}, \beta_{\delta(K)}^{-1} E_{\delta}^{S}\right\}+K^{-s}\right) \\
& \quad\left(\|\breve{\boldsymbol{f}}\|_{H^{s-1}(\breve{\Omega})^{3}}+\|\breve{\boldsymbol{g}}\|_{H^{s+1}(\breve{\Omega})^{3}}\right),
\end{aligned}
$$

where the quantities $\lambda_{\delta}$ and $E_{\delta}^{S}$ are introduced in Proposition 4.1 and Corollary 4.6, respectively.

Condition (4.9) is not at all restrictive and can be avoided when writing more complex estimates. Moreover, if $\lambda_{\delta}$ is bounded independently of $\delta$, which is most often the case, the part of estimate (4.10) concerning the velocity $\breve{\boldsymbol{u}}$ is fully optimal, which is not the case for the mortar spectral element method in general three-dimensional geometries and non-conforming domain decompositions, see [5, Chap VI]. 


\section{Some numerical experiments}

We present numerical tests which would confirm our theoretical predictions in the axisymmetric and general cases. These tests are made on the two types of domains presented in Figure 1, only the first one being convex. Each domain is broken up into rectangles, which enables us to highlight the good convergence properties of the mortar method.

\subsection{The case of axisymmetric data}

We consider the rectangle $\Omega^{a}$ in the left part of Figure 1, broken up into three rectangles $\Omega_{1}^{a}, \Omega_{2}^{a}$, and $\Omega_{3}^{a}$ as follows:

$$
\begin{aligned}
& \left.\Omega^{a}=\right] 0,1[\times]-1,1[; \\
& \left.\quad \Omega_{1}^{a}=\right] \frac{1}{2}, 1[\times]-1, \frac{1}{2}\left[; \quad \Omega_{2}^{a}=\right] 0, \frac{1}{2}[\times]-1, \frac{1}{2}\left[; \quad \Omega_{3}^{a}=\right] 0,1[\times] \frac{1}{2}, 1[.
\end{aligned}
$$

For the first series of tests, we take the axisymmetric data given by

$$
f_{r}(r, z)=1, \quad f_{z}(r, z)=0,
$$

$$
g_{r}(r, z)=r^{7 / 2} z^{2}, \quad g_{z}(r, z)=-\frac{3}{2} r^{5 / 2} z^{3} .
$$
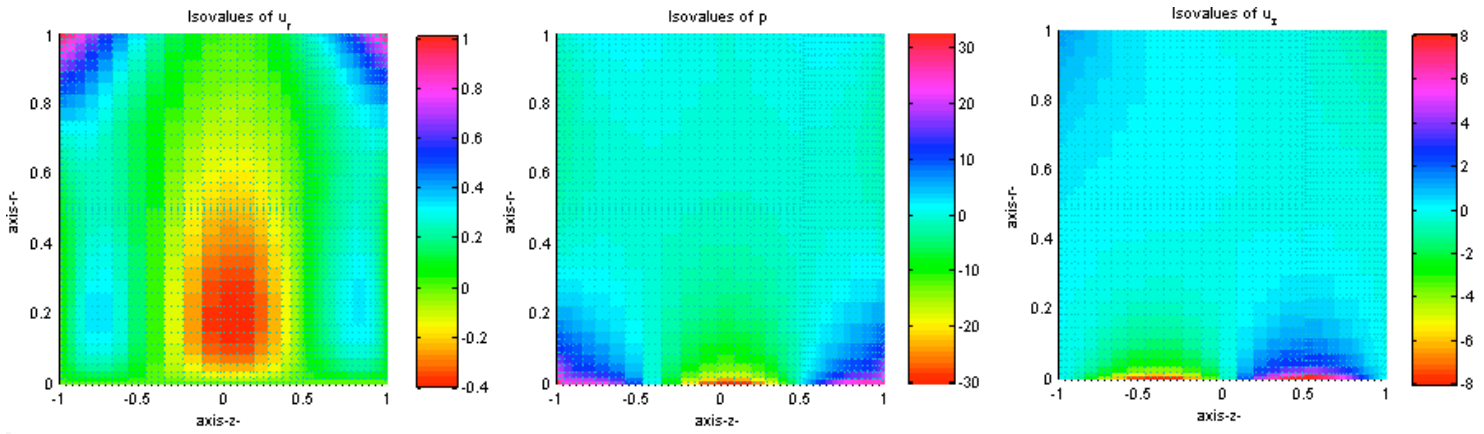

Figure 2: The discrete solution in $\Omega^{a}$ for the data in (5.2)

In Figure 2 we represent the isovalue curves of $u_{r, \delta}, u_{z, \delta}$ and $p_{\delta}$ obtained with

$$
N_{1}=N_{2}=20 \text { and } \quad N_{3}=22 .
$$

The lack of continuity through the interfaces of the three subdomains is not visible on the figure.

We now consider the following singular functions:

$$
u_{r}(r, z)=r^{7 / 2} z^{2}, \quad u_{z}(r, z)=-\frac{3}{2} r^{5 / 2} z^{3}, \quad p(r, z)=r^{1 / 2},
$$


we compute the associated data and finally the discrete solution for these data. The curves in the left part of Figure 3 present the quantities $\log _{10}\left\|\boldsymbol{u}-\boldsymbol{u}_{\delta}\right\|_{L_{1}^{2}\left(\Omega^{a}\right)^{2}}$ (blue line), $\log _{10}\left\|\boldsymbol{u}-\boldsymbol{u}_{\delta}\right\|_{\boldsymbol{Z}}$ (green line) and $\log _{10}\left\|p-p_{\delta}\right\|_{L_{1}^{2}\left(\Omega^{a}\right)}$ (red line) as functions of $\log _{10}(N)$.
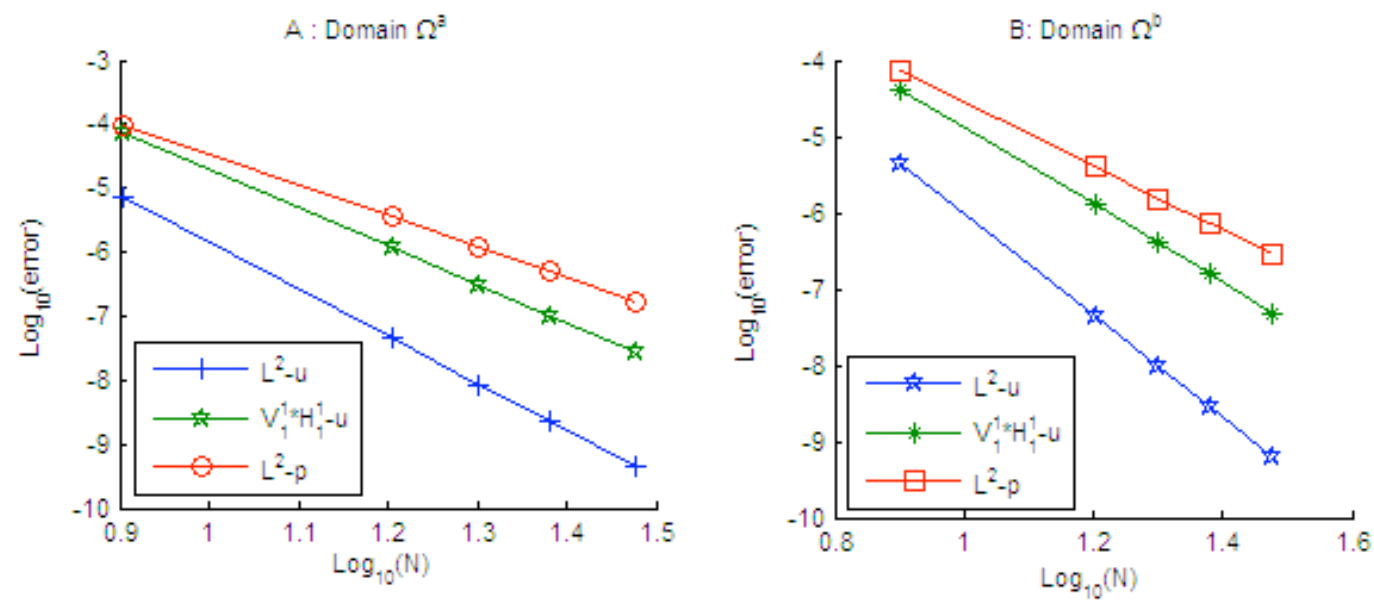

Figure 3: Error curves for the solutions in (5.3) and (5.6)

We now work with the domain $\Omega^{b}$ in the right part of Figure 1, broken up into 5 subdomains, namely

$$
\begin{aligned}
& \left.\Omega^{b}=\right] 0,1[\times]-1,1\left[\backslash \left[\frac{1}{2}, 1\left[\times\left[-\frac{1}{2}, \frac{1}{2}\right],\right.\right.\right. \\
& \left.\Omega_{1}^{b}=\right] \frac{1}{2}, 1[\times]-1,-\frac{1}{2}\left[; \quad \Omega_{2}^{b}=\right] 0, \frac{1}{2}[\times]-1,-\frac{1}{2}\left[; \quad \Omega_{3}^{b}=\right] 0, \frac{1}{2}[\times]-\frac{1}{2}, \frac{1}{2}[, \\
& \left.\Omega_{4}^{b}=\right] 0, \frac{1}{2}[\times] \frac{1}{2}, 1\left[; \quad \Omega_{5}^{b}=\right] \frac{1}{2}, 1[\times] \frac{1}{2}, 1[
\end{aligned}
$$
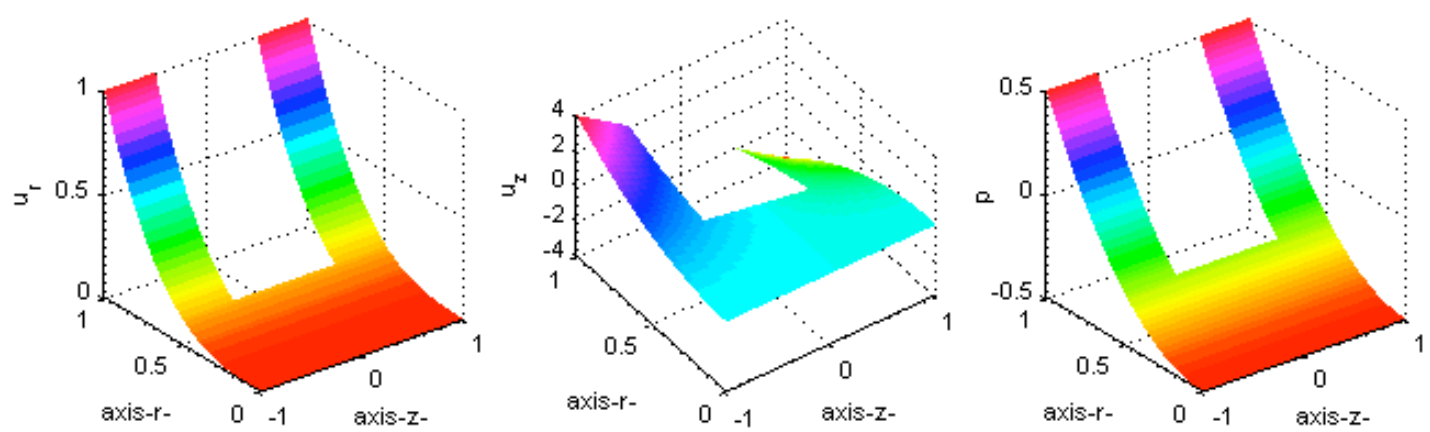

Figure 4: The discrete solution in $\Omega^{b}$ for the data in (5.5)

The (axisymmetric) data are now given by

$$
f_{r}(r, z)=-6 r, \quad f_{z}(r, z)=16 z, \quad \begin{aligned}
& \\
& g_{r}(r, z)=r^{3}, \quad g_{z}(r, z)=-4 r^{2} z .
\end{aligned}
$$


Figure 4 present the layouts of $u_{r, \delta}, u_{z, \delta}$ and $p_{\delta}$ obtained with all $N_{\ell}$ equal to 30.

We consider the following functions in the domain $\breve{\Omega}^{b}$ :

$$
u_{r}(r, z)=r^{7 / 2} z^{2}, \quad u_{z}(r, z)=-3 / 2 r^{5 / 2} z^{3}, \quad p(r, z)=-r,
$$

and as previously compute the associated discrete solution. The curves in the right part of Figure 3 present the quantities $\log _{10}\left\|\boldsymbol{u}-\boldsymbol{u}_{\delta}\right\|_{L_{1}^{2}\left(\Omega^{b}\right)^{2}}$ (blue line), $\log _{10}\left\|\boldsymbol{u}-\boldsymbol{u}_{\delta}\right\|_{\boldsymbol{Z}}$ (green line) and $\log _{10}\left\|p-p_{\delta}\right\|_{L^{2}\left(\Omega^{b}\right)}$ (red line) as functions of $\log _{10}(N)$. It can be noted that, in both parts of this figure, the slope of the curve for the pressure is weaker than for the velocity; this is due to the term $\beta_{\delta}$ in the estimates stated in Theorem 4.4.

\subsection{The general case}
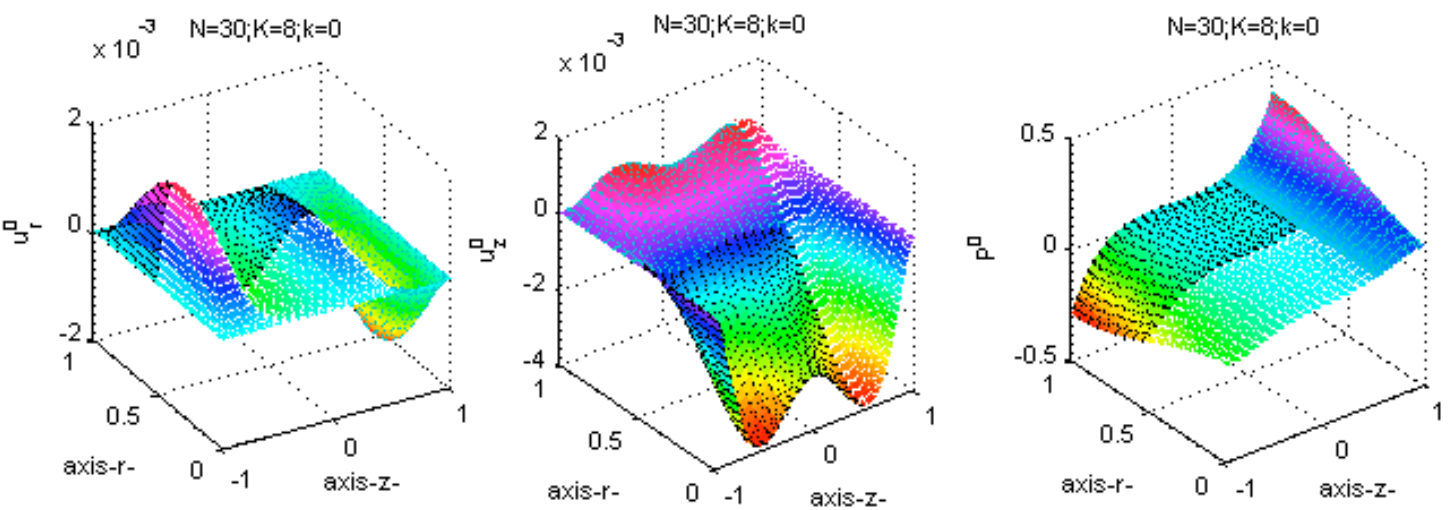

Figure 5: The Fourier coefficients of order 0 of the discrete solution in $\Omega^{a}$ and data in (5.7)
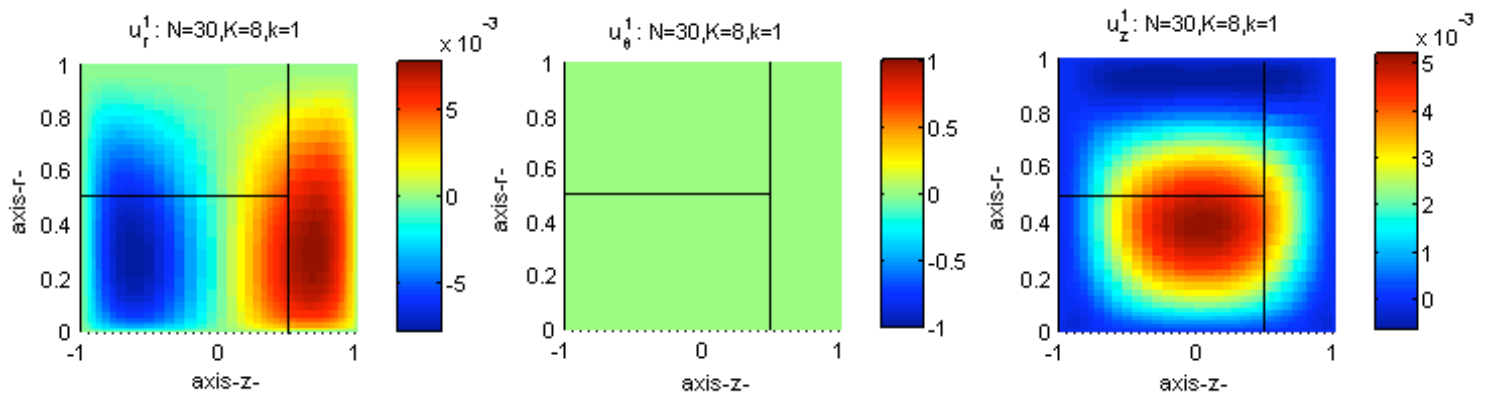

Figure 6: The Fourier coefficients of order 1 of the discrete solution in $\Omega^{a}$ and data in (5.7)

Again in the domain $\Omega^{a}$ defined in (5.1), we firstly work with the data:

$$
\begin{aligned}
&\left(\breve{f}_{r}, \breve{f}_{\theta}, \breve{f}_{z}\right)(r, \theta, z)=\left(r^{3 / 2} z \cos \theta, r^{5 / 2} z \sin \theta, r z^{2} \cos ^{2} \theta\right) \\
&\left(\breve{g}_{r}, \breve{g}_{\theta}, \breve{g}_{z}\right)(r, \theta, z)=(0,0,0) .
\end{aligned}
$$


The layouts of $u_{r, \delta}^{0}, u_{z, \delta}^{0}$ and $p_{\delta}^{0}$ obtained with $K=8$ and all the $N_{\ell}$ equal to 30 are presented of Figure 5. And the isovalue curves of $u_{r, \delta}^{1}, u_{z, \delta}^{1}$ and $p_{\delta}^{1}$ for the same values of the discretization parameters are presented in Figure 6.

To study the slope of the error, we consider the following solution:

$$
\begin{aligned}
\breve{u}_{x}(x, y, z)=x^{2} y^{2}, \quad \breve{u}_{y}(x, y, z)=0, \quad \breve{u}_{z}(x, y, z)=-2 x z y^{2} \\
\breve{p}(x, y, z)=\left(x^{2}+y^{2}\right)^{5 / 4}\left(z^{2}-1\right)^{3 / 2}
\end{aligned}
$$

For the corresponding discrete solution, in the left part of Figure 7, we give the curves of $\log _{10}\left\|\breve{\boldsymbol{u}}-\breve{\boldsymbol{u}}_{\delta}\right\|_{L^{2}\left(\breve{\Omega}^{a}\right)^{3}}$ (blue line), $\log _{10}\left\|\breve{\boldsymbol{u}}-\breve{\boldsymbol{u}}_{\delta}\right\|_{H_{D}^{1}\left(\breve{\Omega}^{a}\right)^{3}}$ (green line), and $\log _{10}\left\|\breve{p}-\breve{p}_{\delta}\right\|_{L^{2}\left(\breve{\Omega}^{a}\right)}$ (red line) as functions of $\log _{10}(N)$.
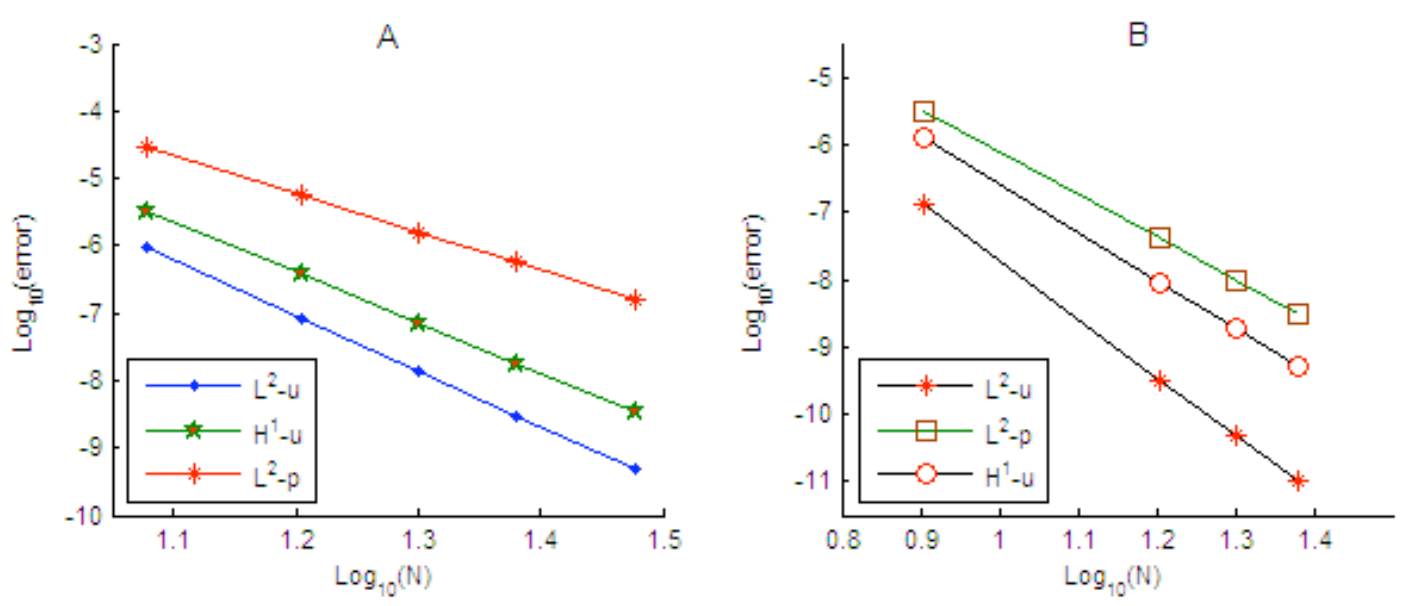

Figure 7: Error curves for the solutions in (5.8) and (5.10)

Finally, we go back to the domain $\Omega^{b}$ defined in (5.4) and we consider the data :

$$
\begin{aligned}
\left(\breve{f}_{x}, \breve{f}_{y}, \breve{f}_{z}\right)(x, y, z)=\left(z^{2}+x^{2}+y^{2},-2 x y,-2 z x\right) & \\
& \left(\breve{g}_{x}, \breve{g}_{y}, \breve{g}_{z}\right)(x, y, z)=\left(0,-x y\left(1-z^{2}\right), 0\right) .
\end{aligned}
$$

We represent in Figure 8 the layouts of $\operatorname{Im}\left(u_{\theta, \delta}^{(1)}\right), p_{\delta}^{(1)}$ and $u_{r, \delta}^{(3)}$ for the discrete solution computed with $K=5$ and all the $N_{\ell}$ equal to 34 . 

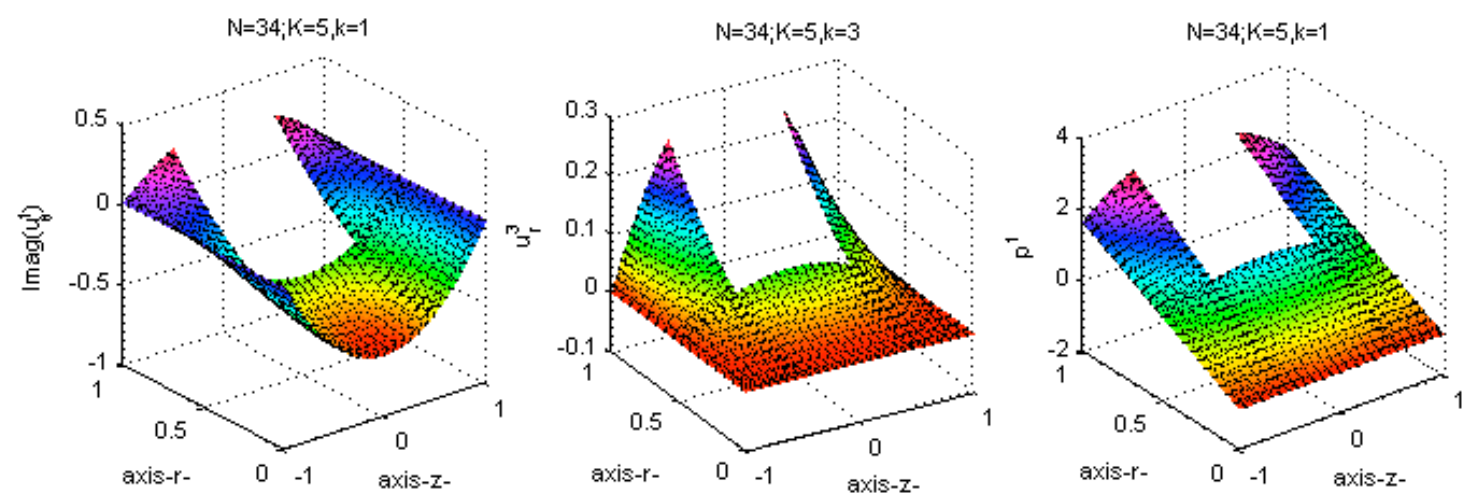

Figure 8: Some Fourier coefficients of the discrete solution in $\Omega^{b}$ and data in (5.9)

Figure 9 illustrates the isovalues of $p_{\delta}^{2}$ and $u_{\theta, \delta}^{2}$ for the discrete solution computed with $K=5$ and all the $N_{\ell}$ equal to 20 .
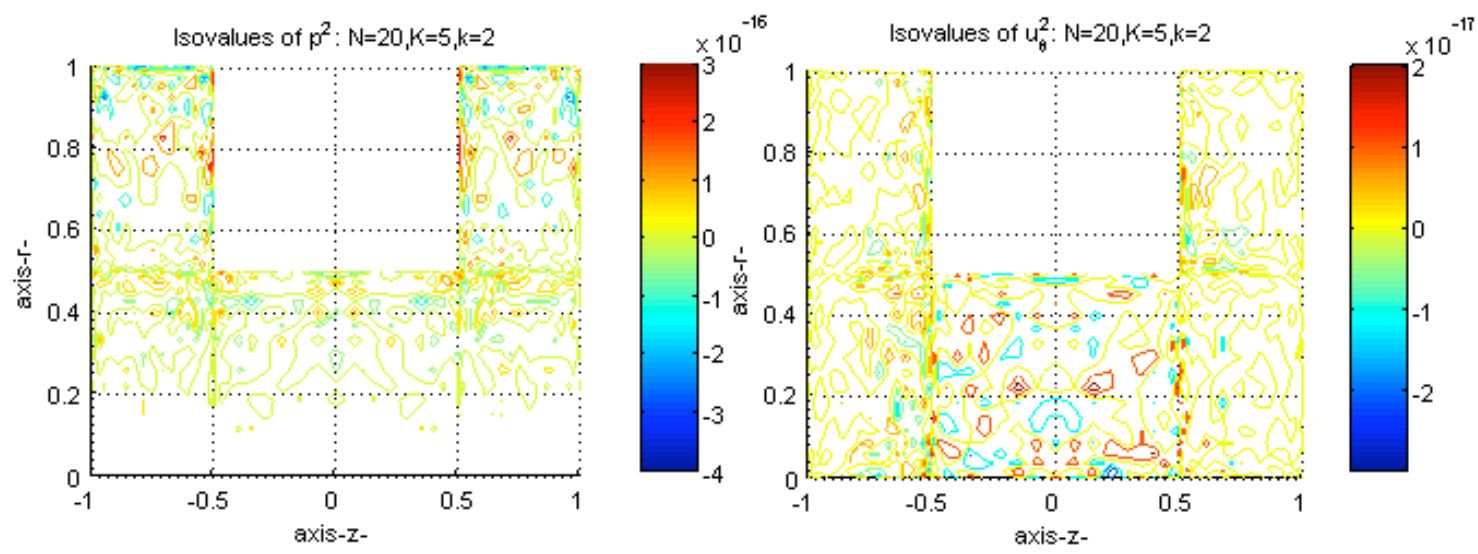

Figure 9: Some Fourier coefficients of the discrete solution in $\Omega^{b}$ and data in (5.9)

To conclude, we consider the solution

$$
\begin{aligned}
& \breve{u}_{x}(x, y, z)=\left(x^{2}+y^{2}\right)^{7 / 3}, \quad \breve{u}_{y}(x, y, z)=0, \\
& \breve{u}_{z}(x, y, z)=-\frac{14}{3}\left(x^{2}+y^{2}\right)^{4 / 3} x, \quad \breve{p}(x, y, z)=x z .
\end{aligned}
$$

We compute the associated discrete solution $\left(\breve{u}_{K, \delta}, \breve{p}_{K, \delta}\right)$. In the right part of Figure 7, we give the curves of the errors $\log _{10}\left\|\breve{\boldsymbol{u}}-\breve{\boldsymbol{u}}_{K, \delta}\right\|_{L^{2}\left(\breve{\Omega}^{b}\right)^{3}}$ (blue line), $\log _{10}\left\|\breve{\boldsymbol{u}}-\breve{\boldsymbol{u}}_{K, \delta}\right\|_{H_{D}^{1}\left(\breve{\Omega}^{b}\right)^{3}}$ (green line), and $\log _{10}\left\|\breve{p}-\breve{p}_{K, \delta}\right\|_{L^{2}\left(\breve{\Omega}^{b}\right)}$ (red line) as functions of $\log _{10}(N)$.

All these results are in good coherence with the estimates proved in Section 4. They confirm the efficiency of our method for solving a three-dimensional problem. 


\section{References}

[1] S.M. Aouadi, J. Satouri - Mortar spectral method in axisymmetric domains, submitted.

[2] M. Azaïez, C. Bernardi, M. Dauge, Y. Maday - Spectral Methods for Axisymetric Domains, "Series in Applied Mathematics" 3, Gauthier-Villars and North-Holland (1999).

[3] Z. Belhachmi - Méthodes d'éléments spectraux avec joints pour la résolution de problèmes d'ordre quatre., Ph.D. Thesis, Université Pierre et Marie Curie, Paris (1994).

[4] Z. Belhachmi, C. Bernardi, S. Deparis, F. Hecht - A truncated Fourier/finite element discretization of the Stokes equations in an axisymmetric domain, Math. Models and Methods in Applied Sciences 16 (2006), 233-263.

[5] C. Bernardi, M. Dauge, Y. Maday - Polynomials in Sobolev Spaces and Application to the Mortar Spectral Element Method, in preparation.

[6] C. Bernardi, Y. Maday - Spectral Methods, Handbook of Numerical Analysis, Vol. V, P.G. Ciarlet and J.L. Lions eds., North-Holland (1996), 209-485.

[7] C. Bernardi, Y. Maday, A.T. Patera - A new nonconforming approach to domain decomposition : the mortar element method, Collège de France Seminar XI, H. Brezis \& J.-L. Lions eds., Pitman (1994), 13-51.

[8] S. Bertoluzza, V. Perrier - The mortar method in the wavelet context, Modél. Math. et Anal. Numér. 35 (2001), 647-673.

[9] J. Boland, R. Nicolaides - Stability of finite elements under divergence constraints, SIAM J. Numer. Anal. 20 (1983), 722-731.

[10] V. Girault, P.-A. Raviart - Finite Element Methods for Navier-Stokes Equations, Theory and Algorithms, Springer-Verlag (1986).

[11] J. Satouri — Méthode d'éléments spectraux avec joints pour des géométries axisymétriques. Thèse de l'Université Pierre and Marie Curie, Paris (2010). 